This is an electronic reprint of the original article. This reprint may differ from the original in pagination and typographic detail.

Author(s): Mansikkamäki, Akseli; Popov, Alexey A.; Deng, Qingming; Iwahara, Naoya; Chibotaru, Liviu F.

Title: Interplay of spin-dependent delocalization and magnetic anisotropy in the ground and excited states of [Gd2@C78]- and [Gd2@C80]-

Year: $\quad 2017$

Version:

Please cite the original version:

Mansikkamäki, A., Popov, A. A., Deng, Q., Iwahara, N., \& Chibotaru, L. F. (2017). Interplay of spin-dependent delocalization and magnetic anisotropy in the ground and excited states of [Gd2@C78]- and [Gd2@C80]-. Journal of Chemical Physics, 147(12), Article 124305. https://doi.org/10.1063/1.5004183

All material supplied via JYX is protected by copyright and other intellectual property rights, and duplication or sale of all or part of any of the repository collections is not permitted, except that material may be duplicated by you for your research use or educational purposes in electronic or print form. You must obtain permission for any other use. Electronic or print copies may not be offered, whether for sale or otherwise to anyone who is not an authorised user. 


\section{Interplay of spin-dependent delocalization and magnetic anisotropy in the ground and excited states of [ $\left.\mathrm{Gd}_{2} @ \mathrm{C}_{78}\right]^{-}$and $\left[\mathrm{Gd}_{2} @ \mathrm{C}_{80}\right]^{-}$}

Akseli Mansikkamäki, Alexey A. Popov, Qingming Deng, Naoya Iwahara, and Liviu F. Chibotaru

Citation: The Journal of Chemical Physics 147, 124305 (2017); doi: 10.1063/1.5004183

View online: http://dx.doi.org/10.1063/1.5004183

View Table of Contents: http://aip.scitation.org/toc/jcp/147/12

Published by the American Institute of Physics

\section{Articles you may be interested in}

Stochastic coupled cluster theory: Efficient sampling of the coupled cluster expansion

The Journal of Chemical Physics 147, 124105 (2017); 10.1063/1.4991795

Communication: Explicitly correlated formalism for second-order single-particle Green's function

The Journal of Chemical Physics 147, 121101 (2017); 10.1063/1.5000916

Connections between variation principles at the interface of wave-function and density-functional theories

The Journal of Chemical Physics 147, 134107 (2017); 10.1063/1.4985883

Production of carbon clusters $\mathrm{C}_{3}$ to $\mathrm{C}_{12}$ with a cryogenic buffer-gas beam source

The Journal of Chemical Physics 147, 124201 (2017); 10.1063/1.4995237

Many-body expansion of the Fock matrix in the fragment molecular orbital method

The Journal of Chemical Physics 147, 104106 (2017); 10.1063/1.5001018

Cold collisions of $\mathrm{SH}^{-}$with He: Potential energy surface and rate coefficients

The Journal of Chemical Physics 147, 124301 (2017); 10.1063/1.4994970

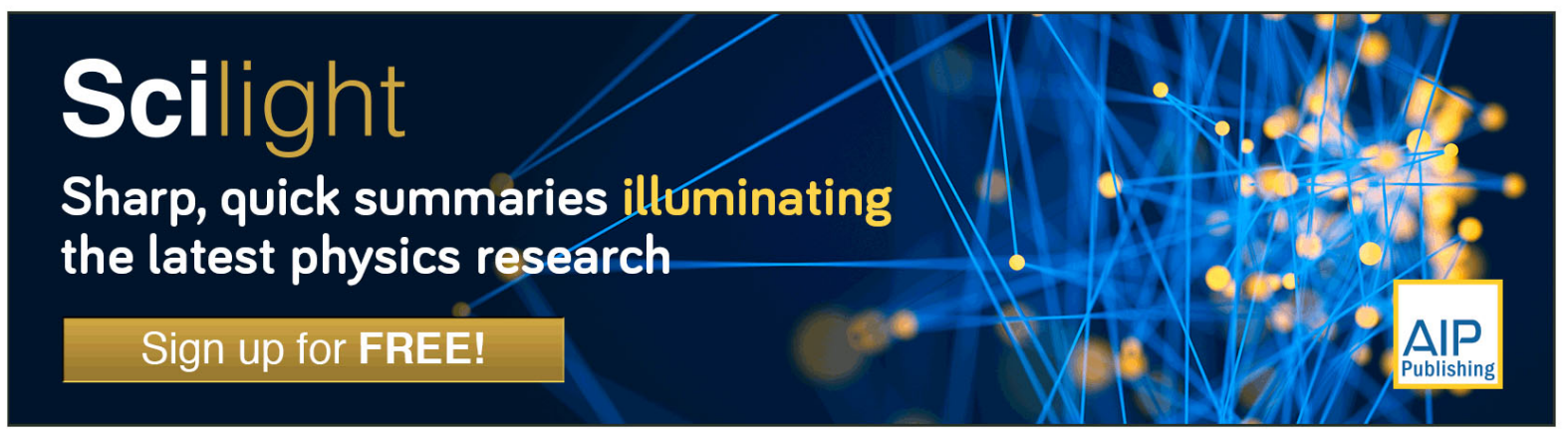




\title{
Interplay of spin-dependent delocalization and magnetic anisotropy in the ground and excited states of $\left[\mathrm{Gd}_{2} @ \mathrm{C}_{78}\right]^{-}$and $\left[\mathrm{Gd}_{2} @ \mathrm{C}_{80}\right]^{-}$
}

\author{
Akseli Mansikkamäki, ${ }^{1,2, a)}$ Alexey A. Popov, ${ }^{3}$ Qingming Deng, ${ }^{3,4}$ Naoya Iwahara, ${ }^{1}$ \\ and Liviu F. Chibotaru, ${ }^{1, b}$ \\ ${ }^{1}$ Theory of Nanomaterials Group, Chemistry Department, Katholieke Universiteit Leuven, Celestijnenlaan 200F, \\ 3001 Leuven, Belgium \\ ${ }^{2}$ Department of Chemistry, Nanoscience Center, University of Jyväskylä, P.O. Box 35, Jyväskylä FI-40014, \\ Finland \\ ${ }^{3}$ Leibniz Institute for Solid State and Materials Research Dresden, Helmholtzstrasse 20, Dresden 01069, Germany \\ ${ }^{4}$ School of Physics and Electronic Electrical Engineering, Huaiyin Normal University, Huai'an 223001, China
}

(Received 22 May 2017; accepted 11 September 2017; published online 27 September 2017)

\begin{abstract}
The magnetic properties and electronic structure of the ground and excited states of two recently characterized endohedral metallo-fullerenes, $\left[\mathrm{Gd}_{2} @ \mathrm{C}_{78}\right]^{-}(\mathbf{1})$ and $\left[\mathrm{Gd}_{2} @ \mathrm{C}_{80}\right]^{-}(\mathbf{2})$, have been studied by theoretical methods. The systems can be considered as $\left[\mathrm{Gd}_{2}\right]^{5+}$ dimers encapsulated in a fullerene cage with the fifteen unpaired electrons ferromagnetically coupled into an $S=15 / 2$ high-spin configuration in the ground state. The microscopic mechanisms governing the $\mathrm{Gd}-\mathrm{Gd}$ interactions leading to the ferromagnetic ground state are examined by a combination of density functional and ab initio calculations and the full energy spectrum of the ground and lowest excited states is constructed by means of $a b$ initio model Hamiltonians. The ground state is characterized by strong electron delocalization bordering on a $\sigma$ type one-electron covalent bond and minor zero-field splitting (ZFS) that is successfully described as a second order spin-orbit coupling effect. We have shown that the observed ferromagnetic interaction originates from Hund's rule coupling and not from the conventional double exchange mechanism. The calculated ZFS parameters of $\mathbf{1}$ and $\mathbf{2}$ in their optimized geometries are in qualitative agreement with experimental EPR results. The higher excited states display less electron delocalization, but at the same time they possess unquenched first-order angular momentum. This leads to strong spin-orbit coupling and highly anisotropic energy spectrum. The analysis of the excited states presented here constitutes the first detailed study of the effects of spin-dependent delocalization in the presence of first order orbital angular momentum and the obtained results can be applied to other mixed valence lanthanide systems. Published by AIP Publishing. https://doi.org/10.1063/1.5004183
\end{abstract}

\section{INTRODUCTION}

Endohedral metallo-fullerenes (EMFs) can stabilize novel chemical species such as small lanthanide clusters by the steric protection and the large electron affinities of the fullerene cages. Such small metal clusters can then exhibit unusual magnetic properties. ${ }^{1-3}$ For example, $\mathrm{LnSc}_{2} \mathrm{~N} @ \mathrm{C}_{80}$ and $\mathrm{Ln}_{2} \mathrm{ScN} @ \mathrm{C}_{80}$ systems ( $\mathrm{Ln}=$ lanthanide) show highly pronounced single-molecule magnet (SMM) behaviour ${ }^{4-6}$ due to the highly axial crystal field around the Ln ions, and $\mathrm{Dy}_{3} \mathrm{~N} @ \mathrm{C}_{80}$ is a geometrically frustrated magnetic molecule with unquenched orbital angular momentum at the Ln sites. $^{7}$

Over the past few years, the magnetic properties of a class of EMFs where $\left[\mathrm{Ln}_{2}\right]^{5+}$ dimers are encapsulated in various fullerene ${ }^{8,9}$ or azafullerene $\left(\mathrm{C}_{79} \mathrm{~N}\right)^{10,11}$ cages have attracted significant attention as some of them exhibit short Ln-Ln distances bordering on a covalent $\mathrm{Ln}-\mathrm{Ln}$ bond ${ }^{12}$ and strong ferromagnetic coupling in their ground spin states. Density

\footnotetext{
a)Electronic mail: akseli.mansikkamaki@jyu.fi

b)Electronic mail: liviu.chibotaru@chem.kuleuven.be
}

functional theory (DFT) calculations on $\mathrm{Gd}_{2} @ \mathrm{C}_{79} \mathrm{~N}$ have shown that the two Gd ions have $4 \mathrm{f}^{7}$ electron configurations and the one "extra" electron occupies a $\sigma$ type orbital with significant contributions from $5 \mathrm{~d}, 6 \mathrm{~s}$, and $6 \mathrm{p}$ orbitals. ${ }^{13,14}$ The DFT calculations also show extensive delocalization of the $\sigma$ electron over the two Gd ions and predict strong ferromagnetic exchange interaction leading to an overall $S=15 / 2$ spin state. At a glance, the ferromagnetic exchange interaction seems to be explained by a double exchange mechanism ${ }^{15-17}$ where a $5 \mathrm{~d}$ electron is resonating between the two $\mathrm{Gd}$ ions and is locally coupled to the spins of the Gd $4 \mathrm{f}$ electrons. An adequate description, however, is not trivial a priori because the electron delocalization coexists with significant spin-orbit coupling (SOC) in the $5 \mathrm{~d}$ orbitals of the $\mathrm{Gd}$ ions. To our knowledge, the double exchange interaction in the presence of strong magnetic anisotropy on the magnetic sites has not been addressed so far.

In the present work, we have conducted a theoretical study on the ground and low-lying excited states of two recently characterized ferromagnetic EMFs, $\left[\mathrm{Gd}_{2} @ \mathrm{C}_{78}\right]^{-}(\mathbf{1})$ and $\left[\mathrm{Gd}_{2} @ \mathrm{C}_{80}\right]^{-}(\mathbf{2}),{ }^{8}$ the latter of which is isoelectronic to $\mathrm{Gd}_{2} @ \mathrm{C}_{79} \mathrm{~N}$. The low-temperature EPR spectra of these systems shows that they both have ferromagnetically coupled 
high-spin $S=15 / 2$ ground states which show some anisotropy. ${ }^{8}$ We will analyze the exchange interactions leading to the ferromagnetic ground state and will explain the ground state anisotropy as a second order effect. We will then proceed to discuss the spectrum of the excited states where an electron is promoted to a $\pi$ or $\delta$ symmetric orbital. Due to the strong Gd-Gd interactions imposed by the short interionic distances and large SOC constant of the Gd ions, the spectrum of the excited states is shaped both by strong exchange interactions due to electron delocalization and by strong spin-orbit coupling. The work presented here will also form a solid foundation for the study of more complicated mixed valence lanthanide systems such as Dy analogues of the systems studied here.

\section{COMPUTATIONAL DETAILS}

\section{A. DFT calculations}

Geometries of $\mathbf{1}$ and $\mathbf{2}$ were optimized at the DFT level using the PBE0 hybrid exchange correlation (XC) functional. ${ }^{18-21}$ Ahlrichs' TZVP $\{62111 / 411 / 1\}$ basis set ${ }^{22}$ was used for the carbon atoms and the ECP53MWB effective core potential of Dolg et al. $^{23}$ with $\{311111 / 31111 / 21111 / 111 / 11\}$ valence part was used for the Gd ions. ${ }^{24}$

All further DFT calculations were carried out on the optimized geometries using all-electron basis sets. Scalar relativistic effects were treated with the zeroth order regular approximation (ZORA) ${ }^{25-27}$ as implemented in OrCA. ${ }^{28}$ The SARCZORA-TZVPP $\quad$ 66111111111111111111/511111111111/ $411111111 / 411 / 111\}$ basis set was used for the Gd ions ${ }^{29}$ and ZORA-def2-SVP $\{511 / 31 / 1\}$ was used for the carbon atoms ${ }^{30}$ along with the corresponding auxiliary basis sets. Energetics of low-lying spin states were probed with broken symmetry (BS) DFT calculations using the PBE0 XC functional. Excitation energies into higher-lying states and the splitting of terms under SOC were calculated using the DFT restricted open-shell configuration interaction singles (DFT/ROCIS) method. ${ }^{31,32}$ The PBE0 XC functional was used along with the default set of parameters for the scaling of the CI matrix elements in the DFT/ROCIS procedure. The CI matrix was constructed by allowing excitations from the singly occupied orbitals into all virtual orbitals up to 5.0 Hartree (which includes almost all virtual orbitals) and the 200 lowest roots were solved for. SOC effects were introduced by constructing the SOC Hamiltonian in a basis of the DFT/ROCIS eigenstates using the spin-orbit mean-field (SOMF) operator ${ }^{33-35}$ and then diagonalizing it to yield the spin-orbit coupled states and eigenvalues. Quasi-restricted orbitals were used in the DFT/ROCIS calculations instead of actual restricted open-shell orbitals to avoid convergence issues in the restricted open-shell KohnSham calculation that would be necessary to produce the true restricted open-shell orbitals. DFT-based ZFS parameters were calculated with the method proposed by Neese ${ }^{36,37}$ using the pure PBE generalized gradient approximation (GGA) functional. ${ }^{18,19}$

All DFT calculations were carried out with the ORCA program suite. ${ }^{38}$ Version 3.0.2 was used for the geometry optimizations, 4.0.0 was used for DFT/ROCIS calculations, and 3.0.3 was used for all other DFT calculations.

\section{B. Multireference ab initio calculations}

Multireference $a b$ initio calculations were first performed on a free $\mathrm{Gd}(\mathrm{II})$ ion. The single ion calculations used the ANORCC-VQZP basis set, which corresponds to a [9s8p6d4f3g2h] contraction. ${ }^{39}$ Scalar relativistic effects were included using the exact two-component (X2C) transformation. ${ }^{40-42} \mathrm{Com}$ plete active space self-consistent field (CASSCF) calculations were carried out using two different active spaces. An $(8,12)$ space was used to calculate the splitting between the lowest Hund and non-Hund states arising from a $4 \mathrm{f}^{7} 5 \mathrm{~d}^{1}$ configuration, and a larger $(8,16)$ active space was used to evaluate the relative energies of states arising from $4 f^{8}$ configurations as compared with the $4 \mathrm{f}^{7} 5 \mathrm{~d}^{1}$ states. The $(8,12)$ active space included the seven $4 \mathrm{f}$ orbitals and five $5 \mathrm{~d}$ orbitals, and the $(8,16)$ active space also included the $6 \mathrm{~s}$ and $6 \mathrm{p}$ orbitals. A state-averaged CASSCF calculation was carried out to solve the lowest roots in each active space. Five roots with $S=4$ and another five roots with $S=3$ corresponding to the ${ }^{9} D$ and ${ }^{7} D$ terms of the $\operatorname{Gd}(\mathrm{II})$ ion were solved in the $\operatorname{CASSCF}(8,12)$ calculation, and a total of nine $S=4$ and sixteen $S=3$ roots corresponding to the ${ }^{9} \mathrm{D},{ }^{9} \mathrm{~S},{ }^{9} \mathrm{P},{ }^{7} \mathrm{D},{ }^{7} \mathrm{~S},{ }^{7} \mathrm{P}$, and ${ }^{7} \mathrm{~F}$ terms were solved in the $\operatorname{CASSCF}(8,16)$ calculation. In the $\operatorname{CASSCF}(8,16)$ calculation, the orbitals were optimized only for the $S=4$ states and the energies of the $S=3$ states were solved by a single diagonalization of the $\mathrm{CI}$ matrix to prevent the orbital optimization procedure from rotating $6 \mathrm{~d}$ orbitals into the active space due to the double shell effect. The remaining dynamic electron correlation not accounted for in the CASSCF calculations was included as a perturbation correction to the energies using the extended multistate complete active space perturbation theory at second order (XMS-CASPT2). ${ }^{43-46}$ The XMS version of CASPT2 was chosen to avoid unphysical splitting of spatially degenerate states. The XMS-CASPT2 correction was calculated only for the CASSCF eigenvalues and no correction to the wave functions was computed. Finally, SOC was introduced using the restricted active space state interaction (RASSI) methodology. ${ }^{47}$ The SOC Hamiltonian was constructed in a basis of spin-free states using the atomic mean field integral (AMFI) operator ${ }^{35,48}$ and then diagonalized to yield the final spin-orbit coupled states.

CASSCF calculations were also carried out on the full systems $\mathbf{1}$ and $\mathbf{2}$ using an ANO-RCC-VTZP basis ([8s7p5d3f2g1h] contraction) for the Gd ions and an ANORCC-VDZP basis ([3s2p1d] contraction) for the carbon atoms. ${ }^{39,49}$ A minimal $(15,15)$ active space was used, which included all $144 \mathrm{f}$ orbital combinations and a $\sigma$ bonding orbital. One $S=15 / 2$, two $S=13 / 2$, two $S=11 / 2$, two $S=9 / 2$, two $S=7 / 2$, two $S=5 / 2$, two $S=3 / 2$, and two $S=1 / 2$ states were solved corresponding to the states in the lowest Hund and non-Hund exchange states in the $\Sigma$ manifolds.

All multireference calculations were carried out with the Molcas quantum chemistry program. ${ }^{50}$ The 8.1 development version was used for the XMS-CASPT2 calculations, and the rest of the calculations were carried out using the 8.0 release version. All Molcas calculations utilized the Cholesky decomposition using a threshold of $10^{-8}$. 


\section{RESULTS AND DISCUSSION}

\section{A. Geometry optimization}

The geometries of $\mathbf{1}$ and $\mathbf{2}$ were fully optimized at the DFT level. Details of the optimization procedures are given in Sec. I of the supplementary material, and the optimized geometries are presented in Fig. 1.1 retains the $D_{3 h}$ symmetry of an elongated $\mathrm{C}_{78}-D_{3 h}(5)$ fullerene cage, whereas in $\mathbf{2}$, the presence of the Gd ions lowers the symmetry of the $\mathrm{C}_{80}-I_{h}$ cage to an approximate $D_{2 h}$ symmetry. The symmetry of the latter agrees with that of $\mathrm{La}_{2} @ \mathrm{C}_{80}$, which has been determined by EPR measurements. ${ }^{51}$ The Gd-Gd distances in $\mathbf{1}$ and $\mathbf{2}$ are $4.088 \AA$ and $3.874 \AA$, respectively. The latter distance is similar to the La-La distance of $3.71 \AA$ A observed in the crystal structure of a benzyl adduct of $\mathrm{La}_{2} @ \mathrm{C}_{80}$ which has a cage that is isoelectronic to $\mathrm{C}_{80}{ }^{6-}$. 9 These geometries were used in all subsequent calculations.

\section{B. General features of electronic structure}

To get an overall picture of the electronic structures of 1 and 2, DFT calculations were conducted on the high-spin ( $S=15 / 2)$ states of both systems. In order to separate features of the electronic structure that are attributable purely to the $\left[\mathrm{Gd}_{2}\right]^{5+}$ core moiety and to those that result from the interaction with the $\mathrm{C}_{78 / 80}{ }^{6-}$ cage, the calculations were also performed on two simple $\left[\mathrm{Gd}_{2}\right]^{5+}$ dimers $\mathbf{1}^{\prime}$ and $\mathbf{2}^{\prime}$ that contain only the Gd ions fixed at the same distance as in the respective caged structures $\mathbf{1}$ and $\mathbf{2}$.

The Kohn-Sham (KS) orbitals of $\mathbf{1}$ and $\mathbf{2}$ are largely similar to each other, and the orbitals of $\mathbf{2}$ closely resemble those that have been reported earlier for the isoelectronic $\mathrm{Gd}_{2} @ \mathrm{C}_{79} \mathrm{~N}$ system. ${ }^{14}$ The fourteen unpaired $4 \mathrm{f}$ electrons occupy seven bonding and seven anti-bonding combinations of the $\mathrm{Gd} 4 \mathrm{f}$ orbitals. These orbitals have only minor contributions from other Gd orbitals or from the cage orbitals and, thus, to a
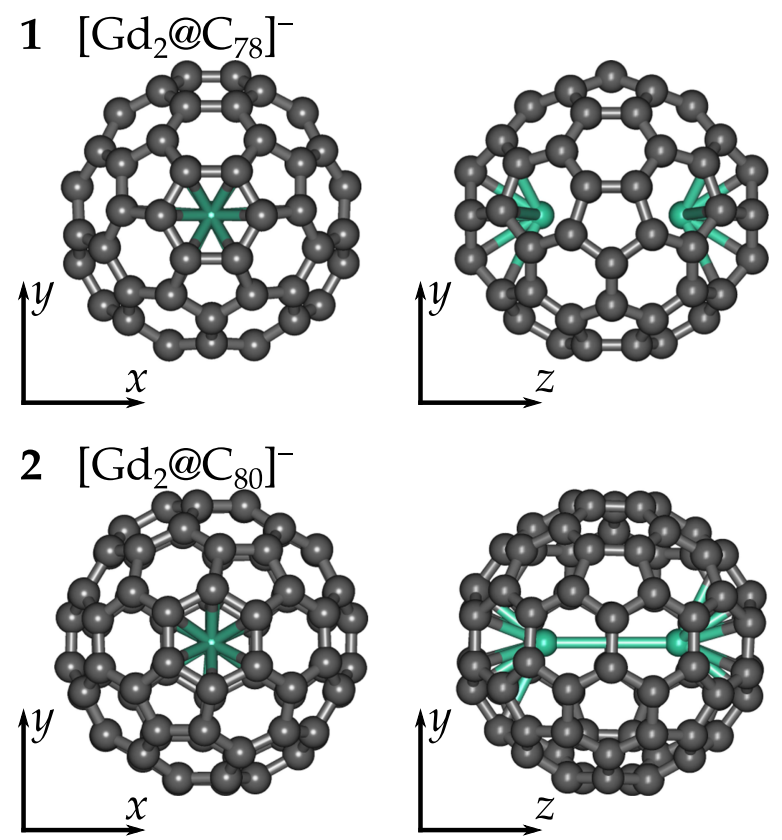

FIG. 1. Optimized geometries of $\mathbf{1}$ and $\mathbf{2}$ (black: C, green: Gd). a)

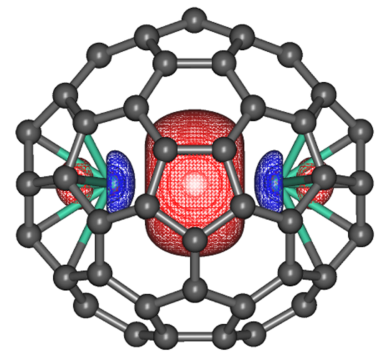

$1\left[\mathrm{Gd}_{2} @ \mathrm{C}_{78}\right]^{-}$

b) $\sigma^{*}$

$\pi^{*}$

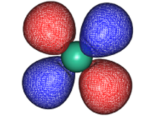

$\delta^{*}$

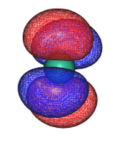

$\delta$

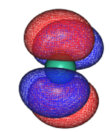

$\pi$

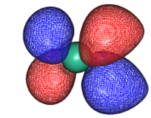

$\sigma$
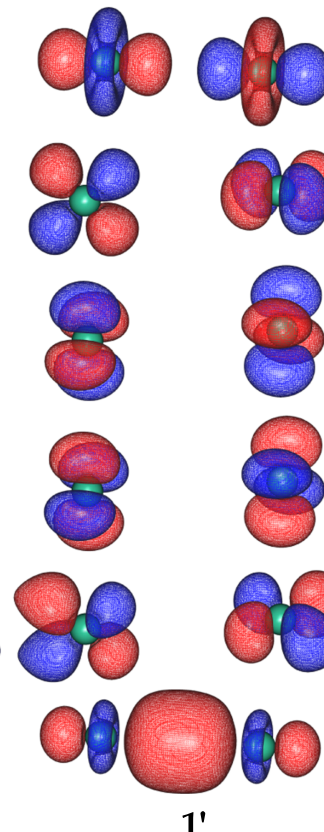

$1^{\prime}$

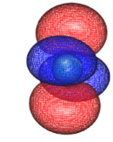

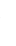

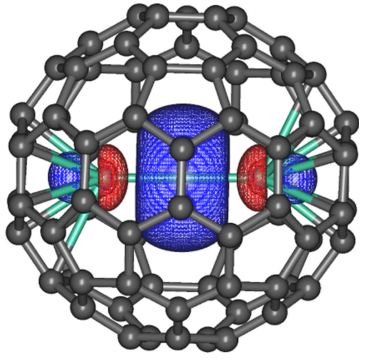

$2\left[\mathrm{Gd}_{2} @ \mathrm{C}_{80}\right]^{-}$
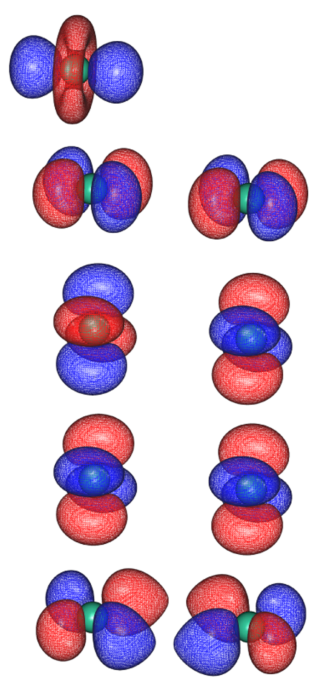

FIG. 2. (a) The $\sigma$-bonding orbital housing the unpaired 5 d electron in $\mathbf{1}$ and $\mathbf{2}$ and (b) the $5 \mathrm{~d}$ orbital combinations in $\mathbf{1}^{\prime}$ (the $5 \mathrm{~d}$ orbital combinations in $\mathbf{2}^{\prime}$ are very similar to the ones presented here).

good approximation, they can be considered as pure $4 \mathrm{f}$ combinations. The one "extra" electron (which will be from here on referred to as the $5 \mathrm{~d}$ electron) occupies a $\sigma$-bonding type orbital with large amplitude in the Gd-Gd bonding region and some minor delocalization into the fullerene cage near the short Gd-C contacts [Fig. 2(a)].

The nature of the $\sigma$ orbital and the related Ln-Ln bonding interaction in analogous systems has been extensively discussed in the literature. ${ }^{12,52,53}$ EPR measurements on related EMFs $\mathrm{Y}_{2} @ \mathrm{C}_{79} \mathrm{~N}$ and $\left[\mathrm{La}_{2} @ \mathrm{C}_{80}\right]^{-}$with empty $4 \mathrm{f}$ shells and one unpaired electron each are consistent with the unpaired spin being localized in the $\left[\mathrm{Ln}_{2}\right]^{5+}$ core. ${ }^{10,51}$ Furthermore, the magnitudes of the hyperfine coupling constants of the ${ }^{139} \mathrm{La}$ and ${ }^{89} \mathrm{Y}$ nuclear spins in the respective systems are relatively large. This suggests that the atomic s orbitals with large amplitudes near the nuclei make a significant contribution to the composition of the $\sigma$ bonding orbitals. In the present case, the $\sigma$ bonding orbitals of 1 and 2 consist mainly of $5 \mathrm{~d}_{\mathrm{z}^{2}}, 6 \mathrm{~s}$, and $6 \mathrm{p}_{\mathrm{z}}$ orbitals. Based on the Löwdin reduced orbital populations, the relative contributions to the orbital composition are $27.0 \%$ from $\mathrm{d}_{\mathrm{z}^{2}}, 24.6 \%$ from $\mathrm{s}, 36.8 \%$ from $\mathrm{p}_{\mathrm{z}}$ and $26.6 \%$ from $\mathrm{d}_{\mathrm{z}^{2}}$, $20.8 \% \mathrm{~s}, 32.6 \% \mathrm{p}_{\mathrm{z}}$ for $\mathbf{1}$ and $\mathbf{2}$, respectively. In order to keep the notation simple and consistent with the description of the virtual orbitals (vide infra), we will from here on refer to the 
$\sigma$ bonding orbital as a combination of the $5 \mathrm{~d}_{\mathrm{z}^{2}}$ orbitals while keeping in mind that in practice the orbitals are combinations of $5 d_{z^{2}}, 6 s$, and $6 p_{z}$ orbitals. Likewise, when we refer to the " $5 \mathrm{~d}$ orbitals," this should be interpreted as the $\pi$ and $\delta$ symmetric $5 \mathrm{~d}$ orbitals and the bonding and anti-bonding $\sigma$ type orbitals.

The virtual 5d orbitals of $\mathbf{1}$ and $\mathbf{2}$ become extremely diffuse and strongly mix with the cage orbitals. In the dimer systems $\mathbf{1}^{\prime}$ and $\mathbf{2}^{\prime}$, however, the virtual $5 \mathrm{~d}$ orbital combinations can be easily identified and they are described in Fig. 2(b). The bonding $\sigma$ symmetric orbitals of $\mathbf{1}^{\prime}$ and $\mathbf{2}^{\prime}$ show similar delocalization and mixing of $\mathrm{s}, \mathrm{p}$, and $\mathrm{d}_{\mathrm{z}^{2}}$ character as the respective orbitals in $\mathbf{1}$ and $\mathbf{2}$. The $\pi$ and $\delta$ symmetric orbitals in $\mathbf{1}^{\prime}$ and $\mathbf{2}^{\prime}$ form two bonding and two anti-bonding combinations (labeled as $\pi^{*}$ and $\delta^{*}$ ) each. The $\pi$ orbitals show significant $\pi$-bonding character, whereas the $\delta$ orbitals are largely non-bonding and confined to their respective Gd ions. Unlike the $\sigma$ bonding orbitals, the respective $\sigma^{*}$ orbitals consist almost purely of the $\operatorname{Gd} 5 \mathrm{~d}_{\mathrm{z}^{2}}$ orbitals.

Energies of the excited $\Sigma, \Pi$, and $\Delta$ terms obtained by $5 \mathrm{~d} \rightarrow 5 \mathrm{~d}$ excitations in $\mathbf{1}, \mathbf{2}, \mathbf{1}^{\prime}$, and $\mathbf{2}^{\prime}$ were calculated with the DFT/ROCIS method and are listed in Table I. For the dimers $\mathbf{1}^{\prime}$ and $\mathbf{2}^{\prime}$, the excitation energies corresponding to various $5 \mathrm{~d} \rightarrow 5 \mathrm{~d}$ excitations can be easily identified by visual examination of the natural transition orbitals. In $\mathbf{1}$ and $\mathbf{2}$, the spectrum of $5 d \rightarrow 5 d$ excitations becomes energetically mixed with the $5 \mathrm{~d} \rightarrow$ cage and cage $\rightarrow$ cage excitations and, therefore, the excited 5d configurations are strongly mixed with excited cage configurations. Because of this, only the lowest $5 \mathrm{~d} \rightarrow 5 \mathrm{~d}$ excitations corresponding to $\sigma \rightarrow \pi$ excitations can be reliably assigned to a transition between two specific electronic configurations. The lowest excitation originating from the $4 \mathrm{f}$ shell lies at much higher energy than the $\sigma \rightarrow \sigma^{*}$ excitation in all systems considered.

The $5 \mathrm{~d} \rightarrow 5 \mathrm{~d}$ excitation energies agree well with what can be qualitatively reasoned from the geometries and orbitals. The covalent interaction between the $\sigma$ symmetric orbitals in the ground state leads to strong stabilization of the ground configuration, and therefore the first excited state lies roughly $20000 \mathrm{~cm}^{-1}$ above the ground state energy. The states in the manifold of excited $5 \mathrm{~d}$ configurations lie much closer together. Under the $D_{3 h}$ symmetry of $\mathbf{1}$, the $\Pi$ and $\Delta$ states should be exactly degenerate. The small splitting of $38 \mathrm{~cm}^{-1}$ between the $\Pi$ states is most likely a result of the fact that the $D_{3 h}$ symmetry was not explicitly imposed on the optimized wave functions. The (pseudo) $D_{2 h}$ symmetry of $\mathbf{2}$ does not conserve

TABLE I. $5 \mathrm{~d} \rightarrow 5 \mathrm{~d}$ excitation energies (in $\mathrm{cm}^{-1}$ ) calculated for $\mathbf{1}, \mathbf{2}, \mathbf{1}^{\prime}$, and $\mathbf{2}^{\prime}$ using the DFT/ROCIS method.

\begin{tabular}{lcccc}
\hline \hline Excitation & $\mathbf{1}$ & $\mathbf{2}$ & $\mathbf{1}^{\prime}$ & $\mathbf{2}^{\prime}$ \\
\hline$\sigma \rightarrow \pi$ & 23293.2 & 21334.8 & 19357.2 & 21237.8 \\
$\sigma \rightarrow \pi$ & 23331.5 & 22910.9 & 19357.2 & 21237.8 \\
$\sigma \rightarrow \delta$ & & & 23630.4 & 26894.6 \\
$\sigma \rightarrow \delta$ & & & 23630.5 & 26894.7 \\
$\sigma \rightarrow \delta^{*}$ & & & 24177.2 & 27733.9 \\
$\sigma \rightarrow \delta^{*}$ & & & 24177.3 & 27734.0 \\
$\sigma \rightarrow \pi^{*}$ & & & 24577.2 & 28532.7 \\
$\sigma \rightarrow \pi^{*}$ & & & 24577.2 & 28532.7 \\
$\sigma \rightarrow \sigma^{*}$ & & & 25115.8 & 29441.0 \\
\hline \hline
\end{tabular}

any degeneracies and a splitting of $1576 \mathrm{~cm}^{-1}$ is observed in the $\Pi$ states.

\section{Magnetic properties of the ground configuration}

In their ground configuration, $\mathbf{1}$ and $\mathbf{2}$ do not have any first order angular momentum because the lone $5 \mathrm{~d}$ electron occupies an orbital of $\sigma$ symmetry which gives rise to a $\Sigma$ term. The orbital interaction is very strong and therefore electron delocalization governs the shape of the spectrum and splits the $\Sigma$ term into bonding and anti-bonding states. The bonding manifold is then further split by exchange interaction into states characterized by some total spin $S$. Thus, the magnetic interaction can be reduced to the isotropic exchange coupling between the two $S_{0, a}=S_{0, b}=S_{0}=7 / 2$ spins arising from the $\mathrm{Gd} 4 \mathrm{f}$ electrons and $s=1 / 2$ of the $5 \mathrm{~d}$ electron. The magnetic anisotropy, which emerges at second order, can be treated as a perturbation at a later stage.

The exchange splitting of the $\Sigma$ terms can be described as a "classical" three-site exchange coupled system where the Gd $4 \mathrm{f}$ electrons form two of the sites and the unpaired $5 \mathrm{~d}$ electron forms the third site. The spin Hamiltonian for this system is written as

$$
\hat{H}_{3 \text {-site }}=-J_{\mathrm{Gd}-\mathrm{Gd}} \hat{\mathbf{S}}_{0, a} \cdot \hat{\mathbf{S}}_{0, b}-J_{\mathrm{Gd}-5 \mathrm{~d}}\left(\hat{\mathbf{s}} \cdot \hat{\mathbf{S}}_{0, a}+\hat{\mathbf{s}} \cdot \hat{\mathbf{S}}_{0, b}\right),
$$

where $\hat{\mathbf{S}}_{0, a}$ and $\hat{\mathbf{S}}_{0, b}$ act on the $S_{0}=7 / 2$ spins of the $4 \mathrm{f}$ electrons at Gd ions $a$ and $b$, respectively, $\hat{\mathbf{s}}$ acts on the spin of the $5 \mathrm{~d}$ electron, and $J_{\mathrm{Gd}-\mathrm{Gd}}$ and $J_{\mathrm{Gd}-5 \mathrm{~d}}$ are the exchange coupling constants.

An alternative approach would be to treat the system as a $\mathrm{Gd}(\mathrm{II}) / \mathrm{Gd}(\mathrm{III})$ mixed valence system with a $5 \mathrm{~d}$ electron resonating between the two Gd ions. This model has been widely used, for example, in the description of $\mathrm{Fe}$ (II)/Fe(III) mixed valence complexes. ${ }^{54}$ However, the $5 \mathrm{~d}$ electron in this model, although highly delocalized, would only have significant amplitude at atomic-like orbitals at the Gd ions and the model would then fail to describe the stabilization of the non-Hund states due to the delocalization of the electron into the bonding region. Therefore, for the description of the ground states, we use the three-site model based on a delocalized $\sigma$ type orbital defined in the Hamiltonian (1). The excited states, where the delocalization due to covalency is greatly reduced because of the much smaller overlap of the $5 \mathrm{~d}$ orbitals, will, however, be interpreted in this manner (see Sec. III F).

In order to evaluate the spectrum of the exchange manifold of the ground configuration, the exchange coupling constants must be determined first. This can be achieved at the DFT level using the broken symmetry (BS) formalism pioneered by Noodleman. ${ }^{55-57}$ Values of the exchange coupling constants extracted from BS DFT calculations are $J_{\mathrm{Gd}-\mathrm{Gd}}$ $=-1.5 \mathrm{~cm}^{-1}, J_{\mathrm{Gd}-5 \mathrm{~d}}=388.6 \mathrm{~cm}^{-1}$ and $J_{\mathrm{Gd}-\mathrm{Gd}}=-1.3 \mathrm{~cm}^{-1}$, $J_{\mathrm{Gd}-5 \mathrm{~d}}=354.2 \mathrm{~cm}^{-1}$ for $\mathbf{1}$ and $\mathbf{2}$, respectively. The coupling constants are similar in magnitude to those calculated for the analogous $\mathrm{Gd}_{2} @ \mathrm{C}_{79} \mathrm{~N}$ system. ${ }^{14}$ Details of the extraction procedure are given in Sec. II B of the supplementary material.

Using the coupling constants obtained with BS DFT calculations and the eigenvalues of Eq. (1) calculated in Sec. II A of the supplementary material, the exchange manifolds of the ground configurations of $\mathbf{1}$ and $\mathbf{2}$ (i.e., $\Sigma$ terms) were constructed and the resulting energy spectrum is presented in Fig. 3 


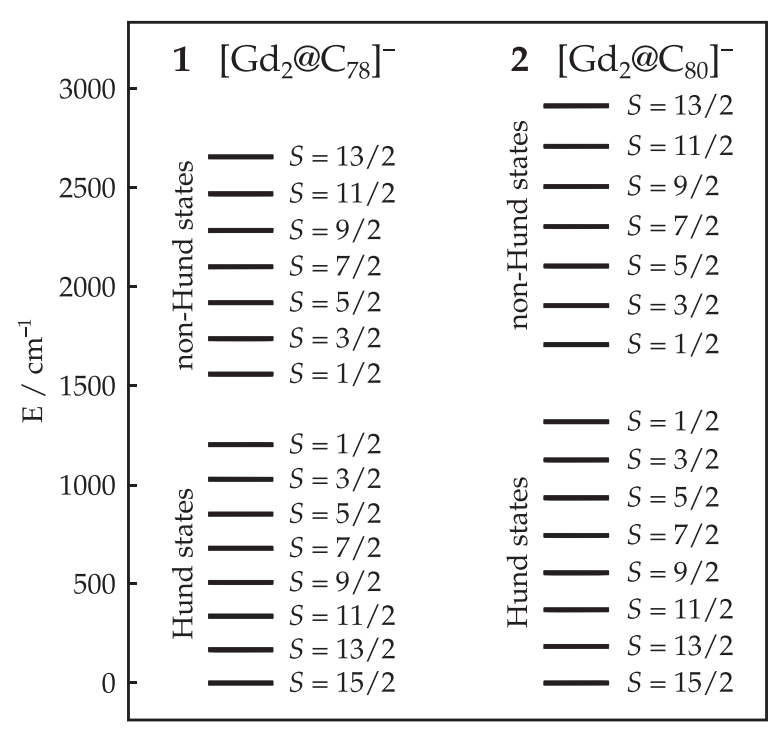

FIG. 3. Energies of the exchange states of the $\sigma$-bonding manifolds of $\mathbf{1}$ and 2 as calculated using Hamiltonian (1) and exchange coupling constants extracted from broken-symmetry DFT calculations.

(numerical values are available in Table S1 of the supplementary material). The low-lying spectrum of the exchange states was also calculated at the CASSCF level for both $\mathbf{1}$ and $\mathbf{2}$ and the results (Table S1 in the supplementary material) agree qualitatively with the BS DFT results. CASPT2 calculations on $\mathbf{1}$ and $\mathbf{2}$ were not possible due to high computational costs, and therefore the CASSCF results do not include any dynamic electron correlation. The exchange coupling constants extracted from BS DFT calculations do include dynamic correlation effects although in an approximate manner. The spectrum constructed from BS DFT results should therefore, in principle, be more accurate than the CASSCF energies and all further discussion will be based on the BS DFT results.

The splitting between the average energies of the Hund and non-Hund manifolds is $1594 \mathrm{~cm}^{-1}$ and $1749 \mathrm{~cm}^{-1}$ for $\mathbf{1}$ and 2 , respectively. The same splitting calculated for a single $\mathrm{Gd}(\mathrm{II})$ ion (see Table III) is $9025 \mathrm{~cm}^{-1}$. Thus, there is a significant reduction of the $4 \mathrm{f}-5 \mathrm{~d}$ Hund's rule coupling strength in $\mathbf{1}$ and 2 as compared with the free ions. This can be explained by the mixing of the $6 \mathrm{~s}$ and $6 \mathrm{p}_{\mathrm{z}}$ orbitals with the $5 \mathrm{~d}_{\mathrm{z}^{2}}$ orbitals due to the lowering of the symmetry by the fullerene cage. The mixing leads to significant delocalization of the $\sigma$ symmetric orbital, as discussed earlier, and to a reduction in the coupling between the unpaired spin in the highly contracted $4 \mathrm{f}$ shell and the $5 \mathrm{~d}$ electron.

Zero-field splitting (ZFS) in the $S=15 / 2$ ground state is weak due to the lack of first order angular momentum in the $\Sigma$ terms but not completely negligible because of the weak mixing of excited configurations into the ground configuration at higher orders of perturbation theory. The experimentally observed EPR spectra measured at $6 \mathrm{~K}$ is consistent with an $S=15 / 2$ spin-state that is weakly split at zero field. The splitting pattern has been modeled by a giant spin Hamiltonian acting on the full $S$ multiplet affording the axial and rhombic ZFS parameters $D$ and $E$, respectively. ${ }^{8}$ The calculated splitting between the ground $\Sigma$ state and the first excited exchange state in 1 and 2 are $168 \mathrm{~cm}^{-1}$ and $184 \mathrm{~cm}^{-1}$, respectively,
TABLE II. The calculated and experimental ZFS parameters of the ground $S=15 / 2$ multiplets of $\mathbf{1}$ and $\mathbf{2}$ in $\mathrm{cm}^{-1}$.

\begin{tabular}{llrcc}
\hline \hline & & DFT & DFT/ROCIS & Experimental $^{8}$ \\
\hline $\mathbf{1}$ & $D$ & -0.233 & 0.297 & 0.0498 \\
& $E$ & 0.000 & 0.000 & 0.00023 \\
$\mathbf{2}$ & $D$ & -0.099 & 0.375 & 0.0339 \\
& $E$ & -0.013 & 0.017 & 0.0102 \\
\hline \hline
\end{tabular}

whereas the experimental ZFS parameters (see Table II) predict the splitting in the ZFS manifolds to be in the range of a few wave numbers. This means that the giant-spin approximation to the ZFS should be well-justified here and, considering the low temperature of the experimental conditions, thermal population of the $S=13 / 2$ exchange states can be safely neglected.

The ZFS parameters were first calculated at the DFT level using the approach proposed by Neese ${ }^{36,37}$ and the values along with the experimentally determined parameters ${ }^{8}$ are listed in Table II. The DFT results predict a negative sign for the $D$ parameter in both $\mathbf{1}$ and $\mathbf{2}$ which is opposite to what is experimentally observed. The negative sign would lead to a completely different electronic structure of the $S=15 / 2$ multiplet than what is experimentally observed. Therefore, the DFT results are clearly incorrect even at a qualitative level. This is not surprising considering that the DFT methods for the treatment of ZFS are known to give highly inaccurate results in a number of cases. ${ }^{58-60}$ The results in Table II were calculated with the pure PBE GGA functional which does not include any exact exchange. Calculations with the hybrid PBE0 functional were also tried but they led to divergence in the coupleperturbed equations. Other DFT based methods ${ }^{61,62}$ for the calculations of ZFS parameters were also attempted but did not lead to any visible improvement.

DFT calculation of ZFS parameters allows one to separate the contributions from SOC and spin-spin coupling (SSC). In the case of lanthanide ions, both SOC and SSC can make a sizable contribution to the ZFS tensor. Whereas the SOC contribution is extremely difficult to calculate at the DFT level, the SSC contributions can be assumed to be more reliable. In the present case, the SSC contributions to the $D$ and $E$ parameters are $D_{S S}=-0.042 \mathrm{~cm}^{-1}, E_{S S}=0.000 \mathrm{~cm}^{-1}$ and $D_{S S}=0.012 \mathrm{~cm}^{-1}, E_{S S}=0.003 \mathrm{~cm}^{-1}$ for 1 and $\mathbf{2}$, respectively. The magnitudes of the SSC parameters are therefore comparable to the SOC contributions. We note in passing that the ZFS parameters calculated purely from the SSC contributions are closer to the experiment than the combined SOC and SSC values but there is no theoretical justification for the neglection of the SOC contribution, and even if that was the case, the sign of the $D_{S S}$ parameter in $\mathbf{1}$ would still be incorrect.

More accurate estimates of the ZFS parameters can, in principle, be obtained by $a b$ initio multireference calculations. However, the ZFS arises from the mixing of a large number of excited states into the ground spin multiplet due to the SOC; these states cannot be generated within some small orbital space outside the complete active space (CAS) used in the CASSCF calculations. Meaningful results could only be obtained by considerably increasing the size of the 
CAS which would then render the calculations computationally untractable. A middle ground between the conventional DFT based methods for the calculations of ZFS parameters and the multireference methods can be reached in the DFT/ROCIS approach that includes SOC in a similar manner to the multireference calculations although using orbitals and orbital energies obtained from restricted open-shell DFT calculations and some empirical scaling parameters. ${ }^{32} \mathrm{~A}$ much larger number of highlying excited states can be accounted with the DFT/ROCIS method as opposed to a CASSCF type calculation. Therefore, we also extracted the ZFS parameters from the energies and eigenvectors produced by the DFT/ROCIS calculation. Details of the extraction process are given in Sec. II C of the supplementary material and the values are listed in Table II. The magnitudes of the values of the $D$ parameters are still an order of magnitude larger than the experimental values, but the sign is reproduced correctly. Considering the extremely small energy differences involved in the calculations and the fact that the energy differences are calculated from the full electronic energies, one is working at the limits of numerical accuracy and no quantitative agreement with experiment should be expected. The main observations made here should be that the sign of the $D$ parameters is positive and that the values are very small compared with the exchange splittings.

The ZFS parameters extracted from the DFT/ROCIS calculation reproduce, in addition to the experimentally observed sign of the $D$ parameter, the main experimentally observed difference between the anisotropies of $\mathbf{1}$ and $\mathbf{2}$ : the considerably larger rhombicity parameter $E$ in $\mathbf{2}$. This can be rationalized based on symmetry considerations. The $D_{3 h}$ symmetry of the geometry of 1 should lead to zero $E$ parameter and the calculated value is indeed exactly zero. The lower pseudo- $D_{2 h}$ symmetry of $\mathbf{2}$ is not high enough to suppress the rhombic components of the anisotropy. The experimentally observed $E$ in $\mathbf{1}$ is, however, very small but non-zero suggesting that under the experimental conditions 1 may show some very minor deviation from the exact $D_{3 h}$ symmetry. It should be noted that the experimental values deviate from zero at the fourth decimal, whereas the calculated parameters cannot be reliably distinguished from numerical noise beyond the third decimal and therefore only three decimals are given in the reported values. The calculated $E$ parameter of $\mathbf{1}$ does indeed deviate from zero at the fourth decimal, but it is impossible to say whether this results from deviation from the $D_{3 h}$ symmetry (which has not been explicitly imposed on the wave function) or simply from numerical noise.

In addition to the $D$ and $E$ parameters that describe the second rank crystal field (CF) parameters, also higher order $\mathrm{CF}$ parameters up to rank 14 (which is the highest relevant rank for a $S=15 / 2$ system) were extracted from the DFT/ROCIS calculation. These are listed in Tables S2 and S3 of the supplementary material. In $\mathbf{1}$, the CF parameters of all ranks above the second are so small that they are either zero or they cannot be reliably distinguished from numerical noise. In the case of $\mathbf{2}$, however, the fourth rank terms are still significant. This also becomes evident by constructing the energies of the zero-field split $S=15 / 2$ manifold by diagonalizing a CF Hamiltonian with various ranks of operators included (see Table S4 and S5 in the supplementary material) and comparing these with the energies obtained by the DFT/ROCIS calculations. It is therefore not necessarily possible to exclude higher rank operators from the spin-Hamiltonian a priori in systems such as $\mathbf{2}$.

\section{Extraction of model Hamiltonian parameters}

In order to adequately describe the low-energy and excited electronic structures, we have derived the effective Hamiltonian describing the systems (vide infra). The parameters used in the construction of the model Hamiltonians were extracted from various ab initio and DFT calculations. The most important parameters governing the low-energy spectrum of an individual Gd(II) ion are the SOC constant $\zeta$ of the $5 \mathrm{~d}$ electrons and the effective Hund's rule coupling parameter $J_{H}^{\prime}$ which determines the energy difference between the Hund states originating from the ${ }^{9} D$ term and the non-Hund states originating from the ${ }^{7} D$ term. The most important interionic parameters that determine the interaction energy between the two ions are the transfer integrals $t_{m_{l}}$ between $5 \mathrm{~d}$ orbitals with orbital angular momentum projection $m_{l}$ on the two Gd ions.

The single-ion parameters were extracted from ab initio calculations performed on a single $\mathrm{Gd}(\mathrm{II})$ ion. The $a b$ initio energies were compared with the eigenvalues of a model Hamiltonian expressed in terms of the parameters $\zeta$ and $J_{H}^{\prime}$,

$$
\hat{H}_{\mathrm{Gd}(\mathrm{II})}=\hat{H}_{\mathrm{SOC}}+\hat{H}_{\mathrm{Hund}}=\zeta \hat{\mathbf{l}} \cdot \hat{\mathbf{s}}-J_{H}^{\prime} \hat{\mathbf{S}}_{0} \cdot \hat{\mathbf{s}},
$$

where $\hat{H}_{\mathrm{SOC}}$ is the SOC Hamiltonian and $\hat{H}_{\text {Hund }}$ is the effective Hund's rule coupling Hamiltonian that is used here in a Heisenberg-like form. ${ }^{63}$

The angular momentum operators $\hat{\mathbf{i}}, \hat{\mathbf{s}}$, and $\hat{\mathbf{S}}_{0}$ act on the orbital angular momentum of the $5 \mathrm{~d}$ electron, the spin of the $5 \mathrm{~d}$ electron, and the total spin of the $4 \mathrm{f}$ electrons, respectively. Detailed derivation of the matrix elements of (2) is presented in Sec. III A of the supplementary material.

Exact expressions of the eigenvalues of $\hat{H}_{\mathrm{Gd}(\mathrm{II})}$ are listed in Table III. Numerical values of $\zeta$ and $J_{H}^{\prime}$ were extracted from the spectrum of $\hat{H}_{\mathrm{Gd}(\mathrm{II})}$ by performing a least squares fit of the energy differences between the ground state and a given excited state to the energy differences between the $a b$ initio calculated states. The fit yielded values $\zeta=1037.84 \mathrm{~cm}^{-1}$ and $J_{H}^{\prime}=2069.53 \mathrm{~cm}^{-1}$. The relative errors compared with the $a b$ initio values are less than $10 \%$ for all states and less than $3 \%$ for more than half of the states. The energies calculated with these parameters, the ab initio values, and experimentally determined values ${ }^{64}$ are also listed in Table III. The calculated values agree very well with the experiment.

The transfer parameters, $t_{m_{l}}$, were extracted from DFT calculations. First a set of Kohn-Sham (KS) orbitals of interest were localized onto the Gd ions so that they maximally resemble their atomic orbital counterparts while still retaining their polarization and hybridization due to the environment. Then, the KS Hamiltonian was transformed into this basis. Within the subspace of the localized orbitals, the transformed KS Hamiltonian has a one-to-one correspondence with a tight-binding Hamiltonian

$$
\begin{aligned}
\hat{H}_{\mathrm{tb}}= & \sum_{\mu, m_{s}} \epsilon_{\mu}\left|a \mu m_{s}\right\rangle\left\langle a \mu m_{s}\left|+\sum_{v, m_{s}} \epsilon_{v}\right| b v m_{s}\right\rangle\left\langle b v m_{s}\right| \\
& +\sum_{\mu, v, m_{s}} t_{\mu \nu}\left(\left|a \mu m_{s}\right\rangle\left\langle b v m_{s}|+| b v m_{s}\right\rangle\left\langle a \mu m_{s}\right|\right),
\end{aligned}
$$


TABLE III. Energies of the spin-orbit coupled total angular momentum $(J)$ states of a Gd(II) ion originating from the ${ }^{9} D$ and ${ }^{7} D$ terms as exact expressions obtained by the diagonalization of the model Hamiltonian (2) and as numerical values obtained by fitting the exact expressions to $a b$ initio values as well as the ab initio calculated and experimental values.

\begin{tabular}{ccccc}
\hline \hline State & Exact expression & Model $\left(\mathrm{cm}^{-1}\right)$ & Ab initio $\left(\mathrm{cm}^{-1}\right)$ & Expt. $^{64}\left(\mathrm{~cm}^{-1}\right)$ \\
\hline${ }^{9} D_{2}$ & $\frac{1}{8}\left(-13 J_{H}^{\prime}-2 \zeta-2 \sqrt{64 J_{H}^{\prime 2}+64 J_{H}^{\prime} \zeta+25 \zeta^{2}}\right)$ & 0 & 0 & 0 \\
${ }^{9} D_{3}$ & $\frac{1}{8}\left(-13 J_{H}^{\prime}-2 \zeta-2 \sqrt{64 J_{H}^{\prime 2}+40 J_{H}^{\prime} \zeta+25 \zeta^{2}}\right)$ & 298 & 317 & 279 \\
${ }^{9} D_{4}$ & $\frac{1}{8}\left(-13 J_{H}^{\prime}-2 \zeta-2 \sqrt{64 J_{H}^{\prime}+8 J_{H}^{\prime} \zeta+25 \zeta^{2}}\right)$ & 758 & 775 & 694 \\
${ }^{9} D_{5}$ & $\frac{1}{8}\left(-13 J_{H}^{\prime}-2 \zeta-2 \sqrt{64 J_{H}^{\prime 2}-32 J_{H}^{\prime} \zeta+25 \zeta^{2}}\right)$ & 1464 & 1425 & 1310 \\
${ }^{9} D_{6}$ & $\frac{1}{8}\left(-29 J_{H}^{\prime}+8 \zeta\right)$ & 2617 & 2393 & 2283 \\
${ }^{7} D_{5}$ & $\frac{1}{8}\left(-13 J_{H}^{\prime}-2 \zeta+2 \sqrt{64 J_{H}^{\prime 2}-32 J_{H}^{\prime} \zeta+25 \zeta^{2}}\right)$ & 9404 & 9045 & 9356 \\
${ }^{7} D_{4}$ & $\frac{1}{8}\left(-13 J_{H}^{\prime}-2 \zeta+2 \sqrt{64 J_{H}^{\prime 2}+8 J_{H}^{\prime} \zeta+25 \zeta^{2}}\right)$ & 9869 & 9695 & 9718 \\
${ }^{7} D_{3}$ & $\frac{1}{8}\left(-13 J_{H}^{\prime}-2 \zeta+2 \sqrt{64 J_{H}^{\prime 2}+40 J_{H}^{\prime} \zeta+25 \zeta^{2}}\right)$ & 10137 & 10153 & 10015 \\
${ }^{7} D_{2}$ & $\frac{1}{8}\left(-13 J_{H}^{\prime}-2 \zeta+2 \sqrt{64 J_{H}^{\prime 2}+64 J_{H}^{\prime} \zeta+25 \zeta^{2}}\right)$ & 10264 & 10470 & 10234 \\
${ }^{7} D_{1}$ & $\frac{1}{8}(3 J+8 \zeta)$ & 10372 & 10672 & 10387 \\
\hline \hline
\end{tabular}

where index $\mu(v)$ runs over all $4 \mathrm{f}$ and $5 \mathrm{~d}$ orbitals on ion $a(b), \epsilon_{\mu}$ and $\epsilon_{\nu}$ are atomic orbital energies, $t_{\mu \nu}$ is the transfer parameter, and $m_{s}$ is the spin projection. The offdiagonal elements in the subspace of the localized orbitals are simply the transfer parameters, and therefore the offdiagonal elements of the KS Fock operator in this basis can be identified as the transfer parameters of the tight-binding Hamiltonian.

The calculations were carried out for orbitals and eigenvalues obtained with both the hybrid PBE0 functional and the pure PBE GGA functional. The exact exchange in the PBE0 functional reduces the delocalization error compared with the pure PBE functional and should therefore offer more accurate results. However, the KS Fock operator constructed using the PBE0 potential includes a contribution from the HatreeFock exchange operator, and therefore the occupied and virtual orbitals do not feel the same potential. This means that, in the case of the PBE0 functional, localization of the $\sigma$ symmetric orbital that would require mixing of occupied and virtual canonical orbitals is not possible without introducing unphysical artifacts, and therefore the $\sigma \leftrightarrow \sigma$ transfer parameters are only available using the GGA functional.

The $4 \mathrm{f}$ orbital combinations are easy to identify, and their localization poses no considerable challenges. Unfortunately, in the case of $\mathbf{1}$ and $\mathbf{2}$, the virtual $5 \mathrm{~d}$ orbitals become strongly mixed with the cage orbitals and isolating a set of $5 \mathrm{~d}$ orbital combinations from the virtual orbital space is not possible without including an arbitrary number of cage, $6 \mathrm{~d}, 6 \mathrm{~s}$, $6 \mathrm{p}$, etc., orbitals into this set. Localization of this arbitrary set would then lead to arbitrary values of transfer parameters which depend on the size of the chosen orbital set and cannot be determined in a unique way. To avoid this problem, the transfer parameters were extracted from the simple $\left[\mathrm{Gd}_{2}\right]^{5+}$ dimers $\mathbf{1}^{\prime}$ and $\mathbf{2}^{\prime}$. The effect of the cage on the direct interaction between the two Gd ions is assumed to be small, and the transfer parameters extracted from calculations on $\mathbf{1}^{\prime}$ and $\mathbf{2}^{\prime}$ should therefore be a good approximation to the respective values in $\mathbf{1}$ and $\mathbf{2}$. The calculated transfer parameters are listed in Table IV as determined with both the PBE0 and PBE functionals. Values extracted from DFT/ROCIS calculations in Sec. III E are also listed in the same table. A complete list of the $5 \mathrm{~d} \leftrightarrow 5 \mathrm{~d}$ and 4f $\leftrightarrow$ 4f parameters calculated at the PBE0 level is given in Tables S6 and S7 of the supplementary material.

The main consequence resulting from neglection of the cage is that the symmetry of both $\mathbf{1}^{\prime}$ and $\mathbf{2}^{\prime}$ is strictly axial and therefore only transfer parameters between $5 \mathrm{~d}$ orbitals corresponding to the same value of $m_{l}$ on the two ions have non-zero values. The values determined with the PBE0 and PBE functionals are very similar to each other with the PBE values being slightly smaller in magnitude. In all subsequent calculations, the PBE0 values will be used but considering the similarity of the values either set should produce comparable results. The explicit value of the $\sigma \leftrightarrow \sigma$ parameter is not needed in any of the calculations beyond knowing that it is larger than the two other parameters.

\section{E. Splitting between $\Sigma$, $\Pi$, and $\Delta$ terms}

Before we discuss the splitting of the $\Pi$ and $\Delta$ terms under the influence of Hund's rule coupling, SOC, and electron

TABLE IV. Transfer parameters between 5d orbitals of the two Gd ions in $\mathbf{1}^{\prime}$ and $\mathbf{2}^{\prime}$ as extracted from DFT calculations using the PBE0 and PBE functionals and as extracted from DFT/ROCIS calculations.

\begin{tabular}{|c|c|c|c|c|c|c|}
\hline & \multicolumn{2}{|c|}{$\operatorname{PBE0}\left(\mathrm{cm}^{-1}\right)$} & \multicolumn{2}{|c|}{$\operatorname{PBE}\left(\mathrm{cm}^{-1}\right)$} & \multicolumn{2}{|c|}{ DFT/ROCIS $\left(\mathrm{cm}^{-1}\right)$} \\
\hline & $t\left(\mathbf{1}^{\prime}\right)$ & $t\left(\mathbf{2}^{\prime}\right)$ & $t\left(\mathbf{1}^{\prime}\right)$ & $t\left(\mathbf{2}^{\prime}\right)$ & $t\left(\mathbf{1}^{\prime}\right)$ & $t\left(\mathbf{2}^{\prime}\right)$ \\
\hline$\sigma \leftrightarrow \sigma$ & & & 9104.2 & 11125.4 & 12557.9 & 14720.5 \\
\hline$\pi \leftrightarrow \pi$ & 2667.8 & 3715.0 & 2275.6 & 3223.1 & 2610.0 & 3647.5 \\
\hline$\pi \leftrightarrow \pi$ & 2667.8 & 3715.0 & 2275.6 & 3223.1 & 2610.0 & 3647.5 \\
\hline$\delta \leftrightarrow \delta$ & 268.1 & 412.8 & 231.6 & 358.4 & 273.4 & 419.7 \\
\hline$\delta \leftrightarrow \delta$ & 268.7 & 412.8 & 231.5 & 358.6 & 273.4 & 419.7 \\
\hline
\end{tabular}


transfer in Sec. III F, we must first determine the relative energies of the energy manifolds arising from the $\Sigma, \Pi$, and $\Delta$ terms. In the $S=15 / 2$ high-spin state, the splitting of the single-ion energy levels due to electron transfer is $\pm t_{m_{l}}$ and therefore the difference between the bonding and anti-bonding states is simply $2 t_{m_{l}}{ }^{65}$ Therefore, energies of the free-ions terms can simply be calculated as the middle point in energy between the respective bonding and anti-bonding states. The transfer parameters extracted from the DFT/ROCIS energy differences are listed in Table IV along with the values determined in Sec. III D. It is clear that the PBE0 and the DFT/ROCIS transfer parameters for the $\pi$ and $\delta$ orbitals are very similar as should be expected.

Setting the energy origin at the $S=15 / 2$ spin-state of the free-ion $\Sigma$ term, the energies of the crystal-field split free-ion $\Pi$ and $\Delta$ terms in their $S=15 / 2$ spin-state are $E_{\Pi}=9409 \mathrm{~cm}^{-1}$, $E_{\Delta}=11346 \mathrm{~cm}^{-1}$ and $E_{\Pi}=10165 \mathrm{~cm}^{-1}, E_{\Delta}=12594 \mathrm{~cm}^{-1}$ for $\mathbf{1}$ and $\mathbf{2}$, respectively.

\section{F. Energy spectrum of the excited $\pi$ and $\delta$ configurations}

Magnetic properties of the excited configurations of $\mathbf{1}$ and 2, where the lone $5 \mathrm{~d}$ electron is promoted to the $\pi$ or $\delta$ orbitals and which give rise to $\Pi$ and $\Delta$ terms, are very different from those of the ground configuration. First, the overlap between the $\pi$ and $\delta$ symmetric $5 \mathrm{~d}$ orbitals on the two ions is much smaller than that between the $\sigma$ symmetric orbitals. This leads to much weaker covalent bonding and thus more localized orbitals and smaller magnitudes of transfer parameters. Second, the orbital angular momentum of the $\pi$ or $\delta$ orbitals is not quenched and first order angular momentum enters the equations. The SOC constant determined earlier for the $\mathrm{Gd}$ (II) ions is $\zeta=1037.84 \mathrm{~cm}^{-1}$ which is roughly of the same order of magnitude as the effective Hund's rule coupling constant $J_{H}^{\prime}=2069.53 \mathrm{~cm}^{-1}$ and the transfer parameters of the $\pi$ or $\delta$ orbitals listed in Table IV. Therefore, all of these interactions must be treated on equivalent footing. The exchange interaction between the $4 \mathrm{f}$ electrons (described by $J_{\mathrm{Gd}-\mathrm{Gd}}$ in the ground configuration) is, however, several orders of magnitude smaller than the other interactions and will be neglected in all subsequent calculations. Under these conditions, the excited states can be viewed as a mixed valence Gd(II)/Gd(III) system and the magnetism can be described in terms of a double exchange model. The general idea of the model is that the $5 \mathrm{~d}$ electron resonates between the two sites where it is coupled to the $4 \mathrm{f}$ spins. This introduces a spin-dependent delocalization into the system. The concept was originally proposed in the context of ionic solids by Zener, ${ }^{15}$ Anderson and Hasegawa, ${ }^{16}$ and de Gennes, ${ }^{17}$ and later adapted to molecular systems by Girerd ${ }^{54,66,67}$ and Noodleman. ${ }^{68}$ Unlike in a conventional treatment of the double exchange, however, the presence of unquenched first-order orbital angular momentum in the present case means that SOC has to be explicitly introduced into the model and the splitting of energy levels will be very different from the spin-only case.

The crystal-field removes the five-fold degeneracy of the orbital $l=2$ states. We will assume a crystal-field with an axial symmetry (trigonal or higher) and, thus, the crystal-field will retain the two-fold orbital degeneracies of the $\Pi$ and $\Delta$ states. In the case of $\mathbf{1}$, this is correct, but for $\mathbf{2}$, this assumption constitutes an approximation. We will base most of our discussion on the strong crystal-field limit where the crystalfield splitting is assumed strong enough so that mixing of the $m_{l}=0$ state into the $m_{l}= \pm 1$ states and $m_{l}= \pm 1$ into $m_{l}= \pm 2$ states by SOC can be neglected. The splitting between the $\Pi$ and $\Delta$ states determined in Sec. III E is $1937 \mathrm{~cm}^{-1}$ and $2429 \mathrm{~cm}^{-1}$ for $\mathbf{1}$ and $\mathbf{2}$, respectively, which is roughly twice the SOC constant and therefore neglecting the $\Pi-\Delta$ mixing is undoubtedly an approximation. The effect of this simplification to the results derived in this section will be discussed later in Sec. III G, and it will be shown that the conclusions made here remain valid even when the mixing is taken into account.

The full Hamiltonian within a given $m_{l}= \pm 1$ or $m_{l} \pm 2$ crystal-field doublet is of the form

$$
\hat{H}_{\text {full }}=\hat{H}_{\text {SOC }}^{a}+\hat{H}_{\text {Hund }}^{a}+\hat{H}_{\text {SOC }}^{b}+\hat{H}_{\text {Hund }}^{b}+\hat{H}_{\text {transfer }},
$$

where the superscripts $a$ and $b$ indicate operators that only act on states where the $5 \mathrm{~d}$ electron is localized at ion $a$ or $b$ and $\hat{H}_{\text {transfer }}$ is the transfer Hamiltonian that couples the localized states. We will first diagonalize the single-ion Hamiltonians $\left(\hat{H}_{\mathrm{SOC}}^{a}+\hat{H}_{\mathrm{Hund}}^{a}\right.$ and $\hat{H}_{\mathrm{SOC}}^{b}+\hat{H}_{\text {Hund }}^{b}$ for ions $a$ and $b$, respectively) to account for SOC and Hund's rule coupling and then consider the resonance stabilization of these states due to the electron delocalization.

Derivation of the matrix elements of the single-ion Hamiltonians and details of the diagonalization procedure are given in Sec. III B of the supplementary material. The eigenvalues are

$$
\begin{aligned}
E_{ \pm}\left(J_{H}^{\prime}, \zeta ; S_{0}, M_{K}, m_{l}\right) \\
=\frac{1}{2} J_{H}^{\prime} \pm \frac{1}{2}\left[J_{H}^{\prime 2} s^{2}\left(2 S_{0}+1\right)^{2}-8 J_{H}^{\prime} \zeta m_{l} s^{2} M_{K}\right. \\
\left.\quad+\zeta^{2} m_{l}^{2} \frac{16 s^{2}\left(S_{0}+s\right)}{\left(2 S_{0}+1\right)^{2}}\right]^{1 / 2},
\end{aligned}
$$

where we have only considered the case where $s=1 / 2$ and $S_{0, a}=S_{0, b} \equiv S_{0}$. The middle term in the square brackets ensures that the states with the same absolute total single-ion angular momentum projection $\left|M_{K}+m_{l}\right|$ are degenerate. This introduces a two-fold degeneracy in the states. The single-ion energies do not depend on the orientation of the $4 \mathrm{f}$ spins of the uncoupled ion which introduced an additional eight-fold degeneracy due to the eight possible projections of the $S_{0, a}$ $=7 / 2$ or $S_{0, b}=7 / 2$ spin of the other ion. Therefore, the total degeneracy of the single-ion eigenstates is 16 . These degeneracies cannot be completely lifted by $\hat{H}_{\text {transfer }}$ as at least double degeneracy must be retained in all states due to Kramers' theorem.

Numerical values of $E_{ \pm}\left(J_{H}^{\prime}, \zeta ; S_{0}, M_{K}, m_{l}\right)$ are listed in Table V. The eigenvectors are linear combinations of a Hund and a non-Hund state corresponding to the same value of $M_{K}$ except for $M_{K}=4$ and $M_{K}=-4$ when there is only one available basis state and the eigenvector consists purely of this state. Splitting of the single-ion energy levels as a function of the $\zeta / J_{H}^{\prime}$ ratio is presented in Fig. 4 for the $m_{l}= \pm 1$ and $m_{l}= \pm 2$ crystal-field doublets. At zero $\zeta$, the Hund and non-Hund states form two degenerate manifolds which are split once the value 
TABLE V. Numerical values of the energies $E_{ \pm}\left(J_{H}^{\prime}, \zeta ; S_{0}, M_{K}, m_{l}\right)\left(\mathrm{in} \mathrm{cm}^{-1}\right)$ of the single-ion Hamiltonians calculated for the $m_{l}= \pm 1$ and $m_{l}= \pm 2$ crystal-field doublets.

\begin{tabular}{cccc}
\hline \hline$M_{J}$ & $E\left(m_{l}= \pm 1\right)$ & $M_{J}$ & $E\left(m_{l}= \pm 2\right)$ \\
\hline \pm 3 & 0 & \pm 2 & 0 \\
\pm 2 & 117 & \pm 1 & 212 \\
\pm 1 & 237 & \pm 0 & 433 \\
\pm 0 & 360 & \pm 1 & 665 \\
\pm 1 & 487 & \pm 2 & 910 \\
\pm 2 & 617 & \pm 3 & 1169 \\
\pm 3 & 752 & \pm 4 & 1447 \\
\pm 4 & 892 & \pm 5 & 1747 \\
\pm 5 & 1038 & \pm 6 & 2076 \\
\pm 4 & 8423 & \pm 5 & 8607 \\
\pm 3 & 8564 & \pm 4 & 8907 \\
\pm 2 & 8699 & \pm 3 & 9185 \\
\pm 1 & 8829 & \pm 2 & 9444 \\
\pm 0 & 8956 & \pm 1 & 9689 \\
\pm 1 & 9079 & \pm 0 & 9921 \\
\pm 2 & 9199 & \pm 1 & 10142 \\
\hline \hline
\end{tabular}

of the $\zeta / J_{H}^{\prime}$ ratio is increased. When $\zeta / J_{H}^{\prime} \lesssim 1$, SOC causes a linear splitting within the manifolds and as the ratio increases further the Hund and non-Hund manifolds become increasingly mixed. At small values of $\zeta / J_{H}^{\prime}$, the lower manifold consists of nine energy states and the higher manifold consists of seven energy states, but once $\zeta / J_{H}^{\prime} \gtrsim 1$, the $M_{J}= \pm 5$ (in the case of the $m_{l}= \pm 1$ crystal-field doublet) and the $M_{J}= \pm 6$ (in the case of the $m_{l}= \pm 2$ doublet) states linearly transfer from the lower manifold to the higher-energy manifold. When the $\zeta / J_{H}^{\prime}$ ratio is further increased, the splitting between the two manifolds tends towards infinity. No energy level crossing takes place at any values of $\zeta / J_{H}^{\prime}$. It should be noted that in the real physical situation, increasing $\zeta$ will also lead to mixing of the $m_{l}= \pm 1$ and $m_{l}= \pm 2$ crystal-field doublets as will be discussed in Sec. III G. Eventually SOC will become of similar magnitude and stronger than the crystal-field splitting and the picture presented in Fig. 4 will break down. In the $m_{l}= \pm 1$ crystal-field doublet, $M_{J}= \pm 3$ states lie lowest in energy, and in the case of the $m_{l}= \pm 2$ doublet, $M_{J}= \pm 2$ energy states are the lowest. If $J_{H}^{\prime} \rightarrow \infty$ (i.e., the coupling between the Hund and non-Hund states can be neglected), the energy of the different $M_{K}$ states in the Hund manifold can be expressed as (see Sec. III B in the supplementary material)

$$
E_{J_{H}^{\prime} \rightarrow \infty}\left(\zeta ; M_{K}, m_{l}\right)=\frac{2 \zeta m_{l} s M_{K}}{2 S_{0}+1},
$$

which clearly shows the linear splitting of different $M_{K}$ states as a function of $\zeta$ observed in Fig. 4 at small values of $\zeta / J_{H}^{\prime}$ ratio when the mixing of the Hund and non-Hund manifolds is negligible.

The transfer Hamiltonian $\hat{H}_{\text {transfer }}$ mixing the single-ion states is of the same form as in the tight-binding Hamiltonian (3). Derivation of its matrix elements is given in Sec. III C of the supplementary material. Due to the assumed axial symmetry, $\hat{H}_{\text {transfer }}$ conserves the total angular momentum projection and the value of $m_{l}$. Therefore, the $\hat{H}_{\text {full }}$ matrix will be blockdiagonal in blocks corresponding to the same values of $M_{K}$ $+M_{0, b}$ or $M_{K}^{\prime}+M_{0, a}^{\prime}$ and $m_{l}$. Although the smallest blocks can be easily diagonalized analytically, the characteristic polynomials of the larger blocks cannot be solved exactly to yield analytical expressions for the eigenvalues. Thus, the energy spectrum of $\hat{H}_{\text {full }}$ was obtained by numerical diagonalization. $\hat{H}_{\text {full }}$ has 128 unique eigenvalues for both crystal-field doublets, each of which is doubly degenerate. The splitting of the $\Pi$ and $\Delta$ single-ion states under the influence of electron transfer is presented in Fig. 5 as a function of the respective transfer parameter.

It is immediately clear from Fig. 5 that the single-ion $\Pi$ and $\Delta$ states become energetically mixed even at relatively small values of $|t|$. In the range $|t| \lesssim 2500 \mathrm{~cm}^{-1}$, the Hund and non-Hund manifolds of the $\Pi$ and $\Delta$ states are clearly separated from each other. In the same range of values, the splitting of the states into bonding and anti-bonding manifolds is also observable in the spectrum with the highest density of states in the top and bottom of the Hund and non-Hund manifolds. Beyond $|t| \sim 3000 \mathrm{~cm}^{-1}$, the interaction approaches that of a covalently bound system and the
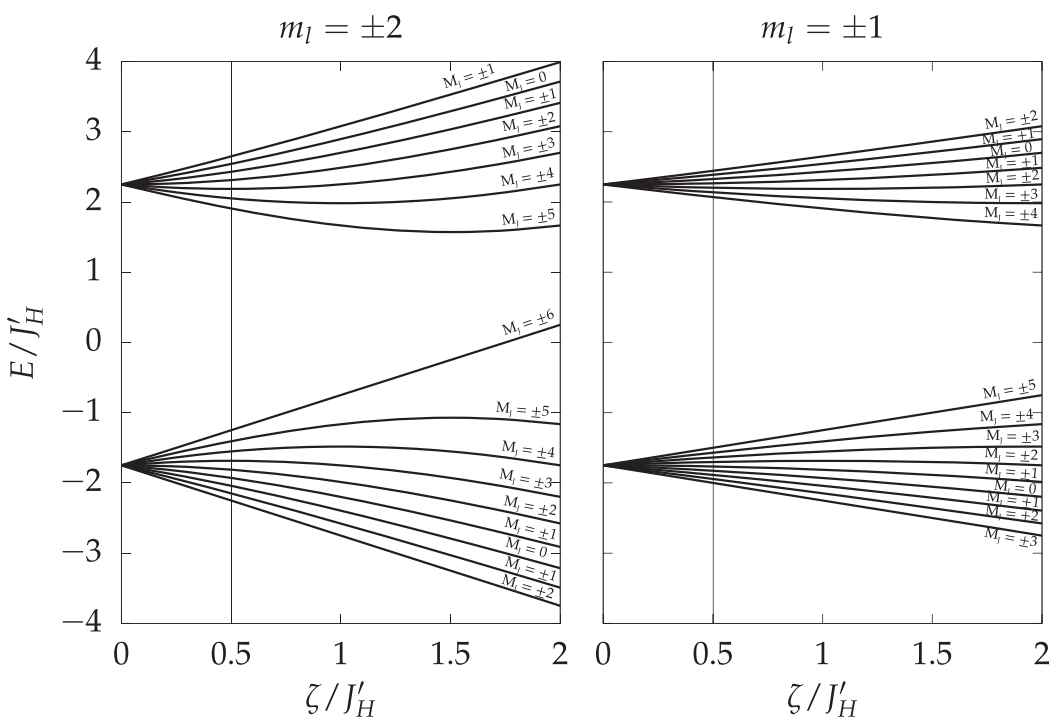

FIG. 4. Effect of the $\zeta / J_{H}^{\prime}$ ratio on the splitting of singleion energy levels within $m_{l}= \pm 2$ and $m_{l}= \pm 1$ crystal field doublets. The vertical lines indicate the $\zeta / J_{H}^{\prime}$ ratio calculated with the $a b$ initio values. 


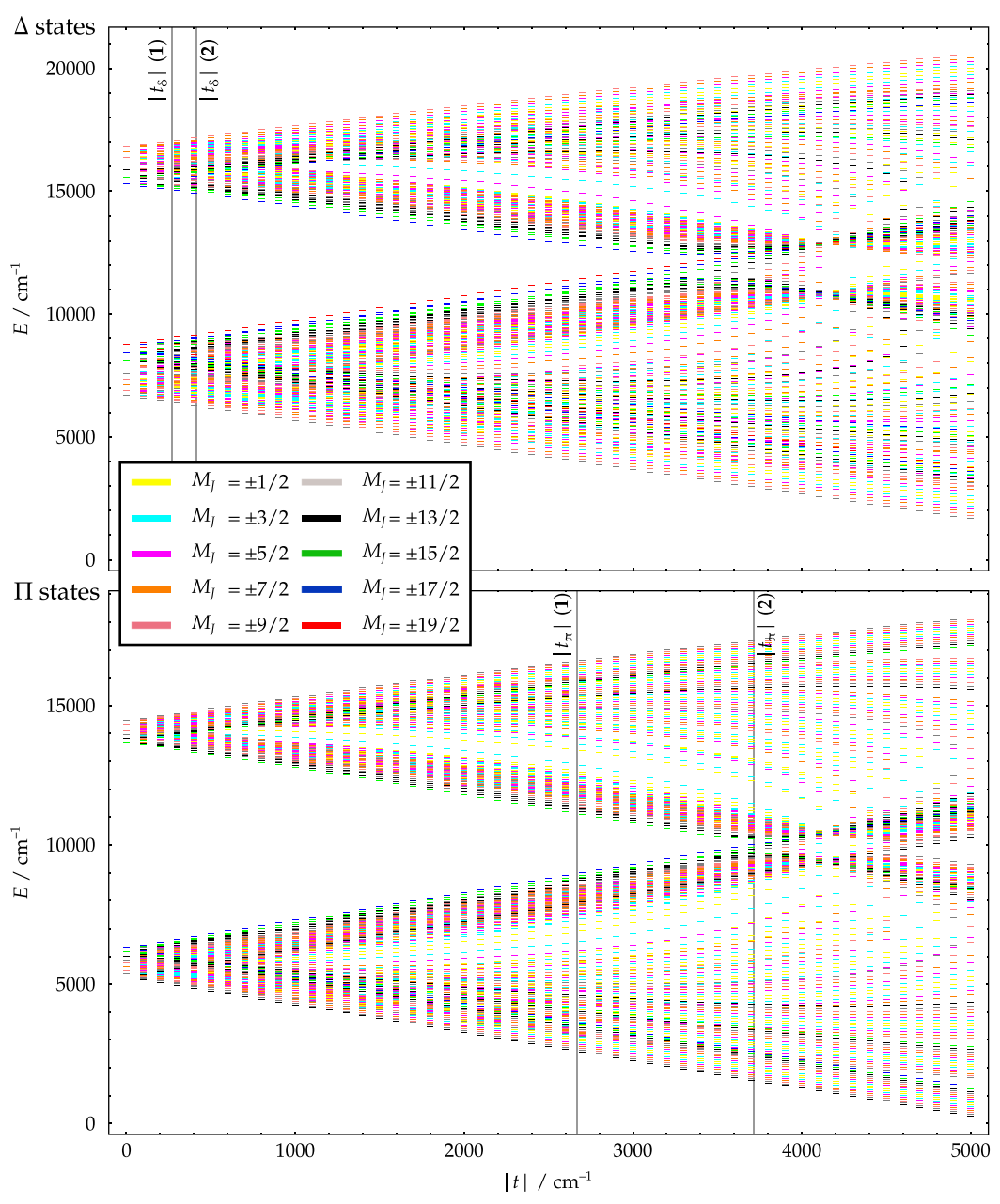

FIG. 5. Splitting of the $\Pi$ (bottom) and $\Delta$ (top) singleion states under the influence of electron transfer in $\mathbf{1}$ and $\mathbf{2}$ as a function of the magnitude of the transfer parameter. The energy levels are color-coded based on the projection of the total angular momentum. Vertical lines indicate the calculated values of the transfer parameters. The energy scale on the vertical axis is that of $\mathbf{1}$; in the case of $\mathbf{2}$, all energy levels are translated to a slightly higher energy due to the larger crystal-field splitting. states in the Hund and non-Hund manifolds with the same value of $M_{J}$ become mixed and all manifolds become energetically intertwined. Considering the $\Delta$ state energy spectrum calculated using the $\delta \leftrightarrow \delta$ transfer parameters of $\mathbf{1}$ and $\mathbf{2}$, the splitting between the Hund and non-Hund manifolds is larger than the transfer-splitting within these manifolds. In the absence of SOC, this situation could be described by the conventional double exchange mechanism. On the other hand, the spectrum of the $\Pi$ states calculated using the $\pi \leftrightarrow \pi$ transfer parameters of $\mathbf{1}$ and $\mathbf{2}$ displays two different situations. In the case of $\mathbf{1}$, the magnitude of the transfer parameter is such that the transfer-splitting is larger than the separation between the Hund and non-Hund manifolds but still small enough to retain a clear splitting between the two manifolds. The transfer parameter of $\mathbf{2}$ is, however, already so large that the interaction is better described as weak covalent interaction than double exchange.

The strong mixing of single-ion states by the transfer interaction makes analysis of the spectrum difficult and it is therefore instructive to consider the case when $J_{H}^{\prime} \rightarrow \infty$ and $|t| \ll \zeta$ where an approximate analytical form can be given for the eigenvalues. Under these conditions, the interaction between single-ion states corresponding to different values of $M_{K}$ can be neglected and conservation of the angular momentum projection under transfer interaction then implies that $M_{0, b}=M_{0, a}^{\prime} \equiv M_{0}$ and $m_{l}=m_{l}^{\prime}$. Eigenvalues within a manifold of states corresponding to a given value of $M_{K}$ (see Sec. III C of the supplementary material) is

$$
\begin{aligned}
E_{ \pm}\left(t ; K, M_{K}, M_{0}, m_{l}\right) & \begin{cases}\frac{2 \zeta m_{l} s M_{K} \pm t\left(K+M_{K}\right)}{2 S_{0}+1} & \text { if } M_{0}=M_{K}-s, \\
\frac{2 \zeta m_{l} s M_{K} \pm t\left(K-M_{K}\right)}{2 S_{0}+1} & \text { if } M_{0}=M_{K}+s, \\
\frac{2 \zeta m_{l} s M_{K}}{2 S_{0}+1} & \text { else. }\end{cases}
\end{aligned}
$$

In the case $M_{J}= \pm 19 / 2$, the expression is exact and in other cases, it is equivalent to the first order perturbation correction to the single-ion energies due to transfer interaction. Figures S2 and S3 of the supplementary material show the splitting of $\Delta$ states in the Hund manifold of $\mathbf{1}$ according to (7) as a function of $|t|$ both in the cases when the coupling between the Hund and non-Hund manifolds is included and when it is neglected. The results show that for large values of $\left|M_{J}\right|$, Eq. (7) describes the splitting reasonably well in the $0<|t|<100 \mathrm{~cm}^{-1}$ range, but for smaller values of $\left|M_{J}\right|$, it is only qualitatively correct.

Equation (7) and Figs. S2 and S3 of the supplementary material show that for a given value of $|t|$ (assuming $|t| \ll \zeta$ ), the transfer splitting is linearly proportional both to $K /\left(2 S_{0}\right.$ $+1)$ and to $M_{K} /\left(2 S_{0}+1\right)$. This is in sharp contrast to the splitting due to the conventional isotropic double exchange mechanism where the splitting is linearly proportional to $(S+s)$ / 
$\left(2 S_{0}+1\right)$, where $S$ is the total spin of the system. ${ }^{65}$ Therefore, in a system with an axial symmetry, the presence of strong anisotropy introduces an Ising-like dependence on the magnitude of the transfer splitting at the strong crystal-field limit.

\section{G. Mixing of states arising from the $\pi$ and $\delta$ configurations}

The crystal-field splitting between the $\Pi$ and $\Delta$ states is, as determined in Sec. III E, $1937 \mathrm{~cm}^{-1}$ for $\mathbf{1}$ and $2429 \mathrm{~cm}^{-1}$ for $\mathbf{1}$. In both cases, this is roughly twice the size of the SOC constant $\left(\zeta=1037.84 \mathrm{~cm}^{-1}\right)$ and therefore some mixing between the $\Pi$ and $\Delta$ terms due to SOC is to be expected. It is, thus, relevant to discuss to what extent this mixing affects the results derived in Sec. III F.

The single-ion Hamiltonian acting in the basis of both the $\Pi$ and $\Delta$ manifolds reads as

$$
\hat{H}_{\text {single-ion }}=\hat{H}_{\mathrm{SOC}}+\hat{H}_{\mathrm{Hund}}+\hat{H}_{\mathrm{CF}, \Delta},
$$

where $\hat{H}_{\mathrm{CF}, \Delta}$ simply adds the crystal-field splitting energy $\Delta E_{\mathrm{CF}}$ to the diagonal elements of the $m_{l}= \pm 2$ states. The matrix elements of $\hat{H}_{\mathrm{SOC}}$ and $\hat{H}_{\mathrm{Hund}}$ can be calculated as derived in Sec. III A of the supplementary material. The characteristic polynomials of the matrix cannot be solved analytically and the matrix can only be diagonalized numerically. The eigenvalues of $\hat{H}_{\text {single-ion }}$ as a function of $\Delta E_{\mathrm{CF}}$ are presented in Fig. 6 along with the eigenvalues calculated using Eq. (5). In the range $E_{\mathrm{CF}}<1.5 \zeta$, the mixing is very strong. Beyond this value, the spectrum is qualitatively similar to that calculated with Eq. (5) with the eigenvalues being slightly shifted in energy. As $E_{\mathrm{CF}}$ is further increased, the approximate values slowly converge towards the exact eigenvalues.

Mixing of the $m_{l}= \pm 1$ states into the $m_{l}= \pm 2$ states means that $M_{K}$ and $m_{l}$ are no longer good quantum numbers. The single-ion states are still characterized by the total angular momentum projection $M_{J}$ and the double degeneracy of the $\left|M_{J}\right|$ states is retained as can be expected as the Kramers degeneracy is not lifted. Therefore, the $\Pi-\Delta$ mixing does not break any degeneracies. All crossings of energy levels belonging to the same value of $\left|M_{J}\right|$ (marked by red circles in Fig. 6) take place at crystal-field splitting $E_{\mathrm{CF}}<1.5 \zeta$. Therefore, all the qualitative changes in the energy level spectrum take place at crystal-field splittings weaker than those determined for $\mathbf{1}$ and $\mathbf{2}$ at the DFT/ROCIS level (1.87 $\zeta$ and $2.24 \zeta$, respectively). Based on this consideration, it is safe to conclude that in the case of $\mathbf{1}$ and $\mathbf{2}$ although the $\Pi-\Delta$ mixing inevitably introduces some error into the eigenvalues calculated in Sec. III F, all the qualitative features of the energy spectrum that can be deduced from the equations given in Sec. III $F$ remain valid even when the $\Pi-\Delta$ mixing is taken into account.

\section{H. Energy spectrum of the excited $4 \mathrm{f}$ configurations}

For the sake of completeness and with possible applications to other $\left[\mathrm{Ln}_{2}\right]^{5+}$ systems in mind, we will also discuss the situation where the "extra" electron occupies a $4 \mathrm{f}$ orbital. In a free $\mathrm{Gd}(\mathrm{II})$ ion, the states arising from the $4 \mathrm{f}^{8}$ configuration are much higher in energy than the states arising from the $4 \mathrm{f}^{7} 5 \mathrm{~d}^{1}$ configuration. Energy of the ${ }^{7} F$ term before the inclusion of

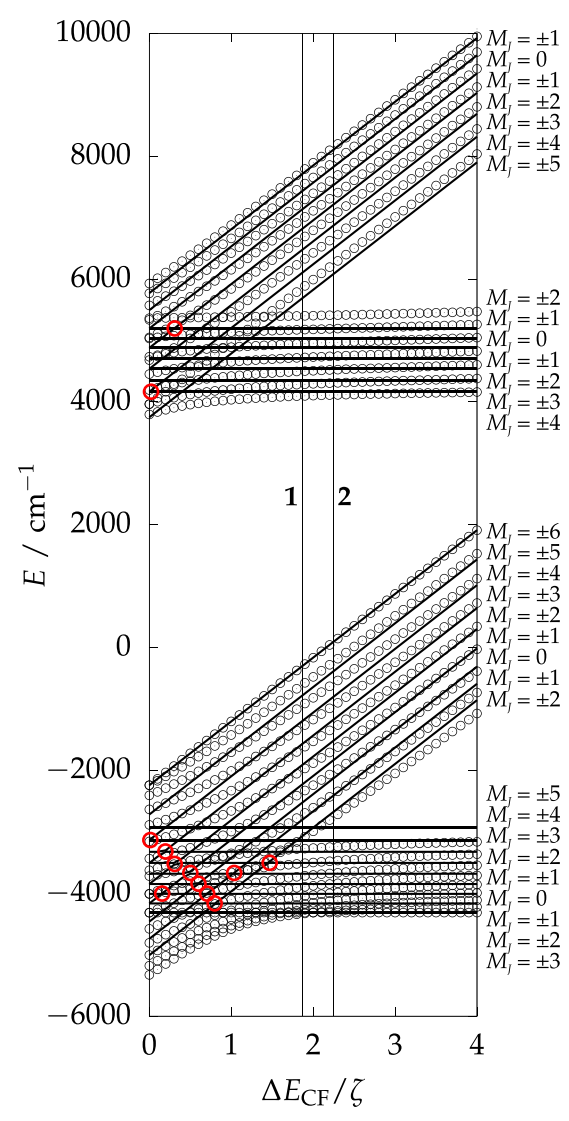

FIG. 6. The single-ion energy levels as a function of the crystal-field splitting between the $\Pi$ and $\Delta$ states $\left(\Delta E_{\mathrm{CF}}\right)$. The solid line describes the splitting when the mixing between $\Pi$ and $\Delta$ states is neglected [Eq. (5)], and black circles indicate the values as calculated by numerical diagonalizition of the single-ion Hamiltonian (8) with the $\Pi-\Delta$ mixing included. Red circles indicate crossings of energy levels [as calculated with (5)] with the same value of $M_{J}$. Vertical lines indicate the values of crystal-field splitting as calculated for $\mathbf{1}$ and $\mathbf{2}$ at the DFT/ROCIS level.

SOC is calculated at the $\operatorname{CASSCF}(8,16) / \mathrm{XMS}-\mathrm{CASPT} 2$ level as $41527 \mathrm{~cm}^{-1}$ compared with the ground state.

We will only consider the Hund configurations where the seven $4 \mathrm{f}$ electrons of a $\mathrm{Gd}(\mathrm{III})$ ion have the same spin and the electron resonating between the ions must then have a different spin. Due to the strong shielding of the $4 \mathrm{f}$ orbitals by $5 \mathrm{~s}$ and $5 p$ orbitals and the large SOC constant of the $4 f$ orbitals, the splitting due to SOC is assumed to be much larger than the crystal-field splitting. Therefore we will first consider the coupling of the $l=3$ orbital angular momentum with the spin $K$ to give a total single-ion angular momentum $J_{0}$. According to Hund's rules (the $4 \mathrm{f}$ shell is more than half-filled), $J_{0}=K$ $+l=6$. As before, we assume an axial crystal field. The crystal field then splits the $\left(2 J_{0}+1\right)$-fold degenerate manifold of states corresponding to different projections $M_{J 0}$ into 6 pairs of doublets characterized by non-zero $\left|M_{J 0}\right|$ and one singlet with $M_{J 0}=0$. When the "extra" electron is localized at ion $a$, this ion is described by $\left|a K l J_{0} M_{J 0}\right\rangle$ and the other ion $b$ is described by $\left|S_{0, b} M_{0, b}\right\rangle$, and hence, in the absence of transfer interaction, the dimer is described by the direct product state $\left|K l J_{0} M_{J 0}\right\rangle \otimes\left|S_{0, b} M_{0, b}\right\rangle$. The opposite situation is expressed by exchanging the indices $a$ and $b$.

When $S_{0, a}=S_{0, b} \equiv S_{0}$, both direct product states are degenerate and each of them has a $\left(2 S_{0}+1\right)$-fold degeneracy 
due to the different values of $M_{0, a}=M_{0, b} \equiv M_{0}$ thus raising the total degeneracy to $4 S_{0}+2$. These states are then mixed by the transfer Hamiltonian $\hat{H}_{\text {transfer }}$. Since the transfer parameters between the $4 \mathrm{f}$ orbitals are small (see Tables S6 and S7 in the supplementary material), whereas the crystal-field is strong due to the short $\mathrm{Gd}-\mathrm{Gd}$ distance, we can neglect the mixing of the crystal-field states corresponding to different values of $\left|M_{J 0}\right|$ by $\hat{H}_{\text {transfer }}$ to a good approximation. Calculation of the matrix elements and details of the diagonalization of $\hat{H}_{\text {transfer }}$ are given in Sec. III D of the supplementary material.

In the present case, the $\mathrm{Gd}(\mathrm{II})$ ion sits in a positive axial crystal-field created by the Gd(III) ion. The Gd(II) ion in its $4 \mathrm{f}^{8}$ configuration is isoelectronic to a $\mathrm{Tb}(\mathrm{III})$ ion and based on purely electrostatic considerations, the lowest single-ion state is the singlet with $M_{J}=0 .{ }^{69}$ The 16 -fold degenerate direct product states involving the $M_{J}=0$ crystal-field state are split into eight Kramers doublets by $\hat{H}_{\text {transfer }}$ (Table VI). The splitting is much weaker than in the case where the Gd(II) ion has a $4 \mathrm{f}^{7} 5 \mathrm{~d}^{1}$ configuration as is expected from the much shorter spatial extent of the $4 \mathrm{f}$ orbitals as compared with the $5 \mathrm{~d}$ orbitals. The largest splitting is obtained for $\left|M_{J 0}\right|=1$ in $\mathbf{2}$, which is still only $25.0 \mathrm{~cm}^{-1}$.

This confirms that the interaction between states characterized by different values of $\left|M_{J 0}\right|$ can be safely neglected when the splitting between the $\left|M_{J 0}\right|$ states is large. In the $M_{J 0}$ $=0$ case, because of the axiality, $\hat{H}_{\text {transfer }}$ merely exchanges the local $M_{J 0}$ and $M_{0}$ projections on the two sites, and the total projection $M_{J 0}+M_{0}$ is conserved. For other values of $M_{J 0}$ in the present system $\left(S_{0}=7 / 2, M_{J 0}\right.$ integer $)$, the same projections are also conserved in the cases when $\left|M_{J 0}\right|>3$. For values $0<\left|M_{J 0}\right| \leq 3$, the $M_{J 0}$ and $-M_{J 0}$ components of the doublets mix and only the total angular momentum projection $M_{J}$ is conserved. In the $M_{J 0}=0$ and $\left|M_{J 0}\right|>3$ cases, the splitting of the various single-ion states is linearly proportional to the transfer parameters and the spacing between the energy levels depends only on the transfer parameters and the angular momentum projections $M_{J 0}$ and $M_{0}$ (see Table VI for $M_{J 0}=0$ and Table S8 of the supplementary material for $\left.\left|M_{J 0}\right|>3\right)$. The transfer interaction is thus purely of Ising type. In the $0<\left|M_{J 0}\right| \leq 3$ case, the interaction is still of Ising

TABLE VI. Splitting of the $M_{J 0}=0$ state arising from the $4 \mathrm{f}^{8}$ configuration in $\mathbf{1}$ and $\mathbf{2}$ due to $4 \mathrm{f} \leftrightarrow 4 \mathrm{f}$ electron transfer.

\begin{tabular}{lccr}
\hline \hline$M_{0}$ & Exact expression & $1\left(\mathrm{~cm}^{-1}\right)$ & $2\left(\mathrm{~cm}^{-1}\right)$ \\
\hline $\pm 1 / 2$ & $-\frac{25}{7392}\left(64 t_{0}+27 t_{1}\right)$ & -8.215 & -13.915 \\
$\pm 3 / 2$ & $-\frac{3}{2464}\left(125 t_{1}+8 t_{2}\right)$ & -3.549 & -6.220 \\
$\pm 5 / 2$ & $-\frac{1}{7392}\left(216 t_{2}+t_{3}\right)$ & -0.105 & -0.185 \\
$\pm 7 / 2$ & $-\frac{1}{1056} t_{3}$ & -0.001 & 0.000 \\
$\pm 7 / 2$ & $\frac{1}{1056} t_{3}$ & 0.001 & 0.000 \\
$\pm 5 / 2$ & $\frac{1}{7392}\left(216 t_{2}+t_{3}\right)$ & 0.105 & 0.185 \\
$\pm 3 / 2$ & $\frac{3}{2464}\left(125 t_{1}+8 t_{2}\right)$ & 3.549 & 6.220 \\
$\pm 1 / 2$ & $\frac{25}{7392}\left(64 t_{0}+27 t_{1}\right)$ & 8.215 & 13.915 \\
\hline \hline
\end{tabular}

type, but the dependence on the transfer parameters becomes more complicated due to the mixing of the states characterized by the $M_{J 0}$ and $-M_{J 0}$ quantum numbers.

\section{GENERAL DISCUSSION AND CONCLUSIONS}

The magnetic properties and the full spectrum of the lowest excited configuration of two endohedral metallo-fullerenes, $\left[\mathrm{Gd}_{2} @ \mathrm{C}_{78}\right]^{-}(\mathbf{1})$ and $\left[\mathrm{Gd}_{2} @ \mathrm{C}_{80}\right]^{-}(\mathbf{2})$, have been studied by theoretical methods. The two $\mathrm{Gd}$ ions have $4 \mathrm{f}^{7}$ configurations with a single "extra" $5 \mathrm{~d}$ electron delocalized between the ions. The spins of the unpaired electrons are coupled to an $S=15 / 2$ high-spin configuration. Depending on whether the $5 \mathrm{~d}$ electron occupies a $\sigma$-symmetric orbital (as in the ground state) or a $\pi$ - or $\delta$-symmetric orbital, the configurations give rise to $\Sigma, \Pi$, or $\Delta$ term, each of which splits very differently under exchange, spin-orbit, and electron transfer interactions leading to a multitude of different energy level structures in the final spectrum.

In the ground $\Sigma$ manifold, the transfer interaction is very strong $t>J_{H}^{\prime}$ bordering on a covalent one-electron $\mathrm{Gd}-\mathrm{Gd}$ bond. Thus, electron delocalization is the dominant effect in the spectrum of the $\Sigma$ states and Hund's rule coupling splits the delocalized states characterized by a total spin $S$. This leads to an energy level spacing which follows the Landé interval rule and the system can be described by a Heisenberg-type Hamiltonian. The interaction between the $4 \mathrm{f}$ spins of the $\mathrm{Gd}$ ions and the $5 \mathrm{~d}$ electron is ferromagnetic and stabilizes the $S$ $=15 / 2$ ground spin state. Due to the lack of spatial degeneracy in the $\Sigma$ states, all first order angular momentum effects are quenched and the total spin $S$ remains a good quantum number. Weak mixing of higher-lying manifolds into the $\Sigma$ states leads to zero-field splitting of the ${ }^{2 S+1} \Sigma$ terms, which can be qualitatively described as a second order effect.

The situation is quite different in the excited $\Pi$ and $\Delta$ states where the $5 \mathrm{~d}$ electron is promoted to a $\pi$ or $\delta$ symmetric orbital and first order angular momentum is not quenched. The splitting of the single-ion states due to Hund's rule coupling is stronger than the splitting due to SOC and therefore the singleion energy levels emerging from the Hund and non-Hund spin configurations retain two distinct manifolds. SOC, however, strongly mixes states corresponding to the same value of the total spin projection of the $\mathrm{Gd}(\mathrm{II})$ ion, and therefore the quantum number $K$ describing the total spin of the ion is no longer a good quantum number. The transfer interaction further mixes the single-ion states and only the total angular momentum projection remains a good quantum number. In both the $\Pi$ and $\Delta$ states, the transfer interaction is stronger than the splitting due to SOC and the single-ion spectra become strongly mixed resulting into a highly complicated energy level structure. In the $\Delta$ states, the splitting due to electron transfer is weaker than the splitting due to Hund's rule coupling. In the absence of SOC, this would be the condition for the validity of the conventional double exchange model. The $\Pi$ states start to approach the covalent limit where the splitting due to the transfer interaction is stronger than the splitting due to Hund's rule coupling. At small magnitudes of the transfer parameter $|t|$, when an analytical expression can be given for the energies of the transfer-split states, it is clear that the splitting 
has a linear dependence on both the total single-ion spin $K$ of the $\mathrm{Gd}$ (II) ion and its projection $M_{K}$. This introduces an Ising-like dependence into the splitting which is very different from the splitting of states in the conventional isotropic double exchange situation where the splitting is proportional only to the total spin $S$ of the coupled system and leads to an energy level spacing proportional to $2 S+1 .{ }^{65}$

The splitting of single-ion energy levels under the electron delocalization was also examined in the case of states arising when the "extra" electron occupies a $4 \mathrm{f}$ orbital combination instead of a $5 \mathrm{~d}$ orbital combination. In this case, the SOC is assumed much stronger than the crystal-field splitting and the Gd(II) single-ion states are characterized by a total angular momentum $J_{0}$ and its projection $M_{J 0}$. Different states corresponding to the same value of $\left|M_{J 0}\right|$ split under the crystal field and the transfer interaction then splits these states. Two distinct cases are observed depending on the magnitude $\left|M_{J 0}\right|$ of the angular momentum projection. If $M_{J 0}$ $=0$ or $\left|M_{J 0}\right|>3$, no mixing between degenerate single-ion states corresponding to values $M_{J 0}$ and $-M_{J 0}$ takes place and the splitting is linearly proportional to the transfer parameters $\left\{t_{m_{l}}\right\}$. If $0<\left|M_{J 0}\right| \leq 3$, the $M_{J 0}$ and $-M_{J 0}$ states become mixed and the dependence of the splitting on $\left\{t_{m_{l}}\right\}$ becomes more complicated. In both cases, the splitting is of Ising type and very weak due to the strongly shielded nature of the 4 f orbitals involved.

The results presented here provide a solid rationalization for the ferromagnetic $S=15 / 2$ ground state of 1 and 2 (and by extension to that of $\mathrm{Gd}_{2} @ \mathrm{C}_{79} \mathrm{~N}$ ) in terms of the microscopic interactions that take place between the two $\mathrm{Gd}$ ions. In addition, we have discussed the magnetism of the excited states arising from configurations where the "extra" electron is promoted to a $\pi$ or $\delta$ symmetric $5 \mathrm{~d}$ orbital combination or to a $4 \mathrm{f}$ orbital combination. These results reveal the unique nature of anisotropic electron transfer interactions. The present work constitutes the first detailed study of spin-dependent delocalization in the presence of firstorder orbital angular momentum. It is worth emphasizing here that in all cases considered here where first-order orbital momentum is involved, the splitting of the energy levels is very different from that associated with conventional double exchange and the splitting will always have a direct dependence on the projection of the angular momenta involved. The derivations presented here can be extended upon to describe the magnetic properties in analogous systems where the orbital contribution to the magnetic moment of the $4 \mathrm{f}$ electrons is not quenched (such as $\left[\mathrm{Dy}_{2} @ \mathrm{C}_{78}\right]^{-}$) as well as to exchange-coupled mixed-valence lanthanide complexes. Therefore the present work also contributes to the future design and understanding of molecules with novel magnetic functionality.

\section{SUPPLEMENTARY MATERIAL}

See supplementary material for the details on geometry optimizations, the extraction of exchange coupling constants and ZFS parameters from broken symmetry DFT and DFT/ROCIS calculations, complete derivations of effective Hamiltonians, complete listings of numerical values of the exchange spectrum arising from the ground configuration, the extracted CF and transfer parameters, the splitting of $\left|M_{J 0}\right|$ states by $4 f \leftrightarrow 4 f$ transfer interaction, and the optimized Cartesian coordinates.

\section{ACKNOWLEDGMENTS}

We would like thank Professor Tatsuhisa Kato (Kyoto University) for sending us his unpublished EPR data and for useful discussions. A.M. gratefully acknowledges computational resources provided by Professor H. M. Tuononen (University of Jyväskylä) and CSC-IT Center for Science, Ltd., in Finland as well as funding provided by the Academy of Finland (Project No. 282499). N.I. is a JSPS Overseas Research Fellow.

${ }^{1}$ J. Zhao, X. Huang, P. Jin, and Z. Chen, Coord. Chem. Rev. 289-290, 315 (2015).

${ }^{2}$ A. A. Popov, S. Yang, and L. Dunsch, Chem. Rev. 113, 5989 (2013).

${ }^{3}$ X. Lu, L. Feng, T. Akasaka, and S. Nagase, Chem. Soc. Rev. 41, 7723 (2012).

${ }^{4}$ R. Westerström, J. Dreiser, C. Piamonteze, M. Muntwiler, S. Weyeneth, H. Brune, S. Rusponi, F. Nolting, A. Popov, S. Yang, L. Dunsch, and T. Greber, J. Am. Chem. Soc. 134, 9840 (2012).

${ }^{5}$ K. Junghans, C. Schlesier, A. Kostanyan, N. A. Samoylova, Q. Deng, M. Rosenkranz, S. Schiemenz, R. Westerström, T. Greber, B. Büchner, and A. A. Popov, Angew. Chem., Int. Ed. 54, 13411 (2015).

${ }^{6}$ D. S. Krylov, F. Liu, S. M. Avdoshenko, L. Spree, B. Weise, A. Waske, A. U. B. Wolter, B. Buchner, and A. A. Popov, Chem. Commun. 53, 7901 (2017).

${ }^{7}$ V. Vieru, L. Ungur, and L. F. Chibotaru, J. Phys. Chem. Lett. 4, 3565 (2013). ${ }^{8}$ T. Yamaguchi, N. Nakatori, T. Mitani, K. Kikuchi, T. Kodama, K. Furukawa, and T. Kato, in The Abstracts of the 50th Fullerenes- Nanotubes- Graphene General Symposium (The University of Tokyo, 2016), p. 43.

${ }^{9}$ L. Bao, M. Chen, C. Pan, T. Yamaguchi, T. Kato, M. M. Olmstead, A. L. Balch, T. Akasaka, and X. Lu, Angew. Chem., Int. Ed. 55, 4242 (2016).

${ }^{10}$ T. Zuo, L. Xu, C. M. Beavers, M. M. Olmstead, W. Fu, T. D. Crawford, A. L. Balch, and H. C. Dorn, J. Am. Chem. Soc. 130, 12992 (2008).

${ }^{11}$ W. Fu, J. Zhang, T. Fuhrer, H. Champion, K. Furukawa, T. Kato, J. E. Mahaney, B. G. Burke, K. A. Williams, K. Walker, C. Dixon, J. Ge, C. Shu, K. Harich, and H. C. Dorn, J. Am. Chem. Soc. 133, 9741 (2011).

${ }^{12}$ A. A. Popov, S. M. Avdoshenko, A. M. Pendás, and L. Dunsch, Chem. Commun. 48, 8031 (2012).

${ }^{13}$ M. K. Singh, N. Yadav, and G. Rajaraman, Chem. Commun. 51, 17732 (2015).

${ }^{14}$ F. Cimpoesu, B. Frecus, C. I. Oprea, H. Ramanantoanina, W. Urland, and C. Daul, Mol. Phys. 113, 1712 (2015).

${ }^{15}$ C. Zener, Phys. Rev. 82, 403 (1951).

${ }^{16}$ P. W. Anderson and H. Hasegawa, Phys. Rev. 100, 675 (1955).

${ }^{17}$ P. G. de Gennes, Phys. Rev. 118, 141 (1960).

${ }^{18}$ J. P. Perdew, K. Burke, and M. Ernzerhof, Phys. Rev. Lett. 77, 3865 (1996).

${ }^{19}$ J. P. Perdew, K. Burke, and M. Ernzerhof, Phys. Rev. Lett. 78, 1396 (1997).

${ }^{20}$ M. Ernzerhof and G. E. Scuseria, J. Chem. Phys. 110, 5029 (1999).

${ }^{21}$ C. Adamo and V. Barone, J. Chem. Phys. 110, 6158 (1999).

${ }^{22}$ A. Schäfer, H. Horn, and R. Ahlrichs, J. Chem. Phys. 97, 2571 (1992).

${ }^{23}$ M. Dolg, H. Stoll, A. Savin, and H. Preuss, Theor. Chim. Acta 75, 173 (1989).

${ }^{24}$ J. Yang and M. Dolg, Theor. Chem. Acc. 113, 212 (2005).

${ }^{25}$ E. van Lenthe, E. J. Baerends, and J. G. Snijders, J. Chem. Phys. 99, 4597 (1993).

${ }^{26}$ E. van Lenthe, E. J. Baerends, and J. G. Snijders, J. Chem. Phys. 101, 9783 (1994).

${ }^{27}$ E. van Lenthe, R. van Leeuwen, E. J. Baerends, and J. G. Snijders, Int. J. Quantum Chem. 57, 281 (1996).

${ }^{28}$ C. van Wüllen, J. Chem. Phys. 109, 392 (1998).

${ }^{29}$ D. A. Pantazis and F. Neese, J. Chem. Theory Comput. 5, 2229 (2009).

${ }^{30}$ D. A. Pantazis, X.-Y. Chen, C. R. Landis, and F. Neese, J. Chem. Theory Comput. 4, 908 (2008).

${ }^{31}$ M. Roemelt and F. Neese, J. Phys. Chem. A 117, 3069 (2013).

${ }^{32}$ M. Roemelt, D. Maganas, S. DeBeer, and F. Neese, J. Chem. Phys. 138, 204101 (2013). 
${ }^{33}$ F. Neese, J. Chem. Phys. 122, 034107 (2005).

${ }^{34}$ A. Berning, M. Schweizer, H.-J. Werner, P. J. Knowles, and P. Palmieri, Mol. Phys. 98, 1823 (2000).

${ }^{35}$ B. A. Heß, C. M. Marian, U. Wahlgren, and O. Gropen, Chem. Phys. Lett. 251, 365 (1996).

${ }^{36}$ F. Neese, J. Am. Chem. Soc. 128, 10213 (2006).

${ }^{37}$ F. Neese, J. Chem. Phys. 127, 164112 (2007).

${ }^{38}$ F. Neese, Wiley Interdiscip. Rev.: Comput. Mol. Sci. 2, 73 (2012).

${ }^{39}$ B. O. Roos, R. Lindh, P.-A. Malmqvist, V. Veryazov, P.-O. Widmark, and A. C. Borin, J. Phys. Chem. A 112, 11431 (2008).

${ }^{40}$ W. Kutzelnigg and W. Liu, J. Chem. Phys. 123, 241102 (2005).

${ }^{41}$ M. Filatov, J. Chem. Phys. 125, 107101 (2006).

${ }^{42}$ D. Peng and M. Reiher, Theor. Chem. Acc. 131(1), 1081 (2012).

${ }^{43}$ B. O. Roos, P. Linse, P. E. Siegbahn, and M. R. Blomberg, Chem. Phys. 66, 197 (1982).

${ }^{44}$ K. Andersson, P. Å. Malmqvist, B. O. Roos, A. J. Sadlej, and K. Wolinski, J. Phys. Chem. 94, 5483 (1990).

${ }^{45}$ K. Andersson, P.-A. Malmqvist, and B. O. Roos, J. Chem. Phys. 96, 1218 (1992).

${ }^{46}$ T. Shiozaki, W. Gyốrffy, P. Celani, and H.-J. Werner, J. Chem. Phys. 135, 081106 (2011).

${ }^{47}$ P. Å. Malmqvist, B. O. Roos, and B. Schimmelpfennig, Chem. Phys. Lett. 357, 230 (2002).

${ }^{48}$ O. Christiansen, J. Gauss, and B. Schimmelpfennig, Phys. Chem. Chem. Phys. 2, 965 (2000).

${ }^{49}$ O. B. Roos, V. Veryazov, and P.-O. Widmark, Theor. Chem. Acc. 111, 345 (2004).

${ }^{50}$ F. Aquilante, J. Autschbach, R. K. Carlson, L. F. Chibotaru, M. G. Delcey, L. De Vico, I. F. Galván, N. Ferré, L. M. Frutos, L. Gagliardi, M. Garavelli, A. Giussani, C. E. Hoyer, G. Li Manni, H. Lischka, D. Ma, P. A.. Malmqvist, T. Müller, A. Nenov, M. Olivucci, T. B. Pedersen, D. Peng, F. Plasser, B. Pritchard, M. Reiher, I. Rivalta, I. Schapiro, J. Segarra-Martí,
M. Stenrup, D. G. Truhlar, L. Ungur, A. Valentini, S. Vancoillie, V. Veryazov, V. P. Vysotskiy, O. Weingart, F. Zapata, and R. Lindh, J. Comput. Chem. 37, 506 (2015)

${ }^{51}$ T. Kato, J. Mol. Struct. 838, 84 (2007).

${ }^{52}$ N. Samoylova, S. Avdoshenko, D. Krylov, H. R. Thompson, A. Kirkhorn, M. Rosenkranz, S. Schiemenz, F. Ziegs, A. Wolter, S. Yang, S. Stevenson, and A. A. Popov, Nanoscale 9, 7977 (2017).

${ }^{53}$ Z. Wang, R. Kitaura, and H. Shinohara, J. Phys. Chem. C 118, 13953 (2014).

${ }^{54}$ G. Blondin and J. J. Girerd, Chem. Rev. 90, 1359 (1990).

${ }^{55}$ L. Noodleman, J. Chem. Phys. 74, 5737 (1981).

${ }^{56}$ L. Noodleman, J. G. Norman, Jr., J. H. Osborne, A. Aizman, and D. A. Case, J. Am. Chem. Soc. 107, 3418 (1985).

${ }^{57}$ L. Noodleman and E. R. Davidson, Chem. Phys. 109, 131 (1986).

${ }^{58}$ M. Atanasov, D. Aravena, E. Suturina, E. Bill, D. Maganas, and F. Neese, Coord. Chem. Rev. 289-290, 177 (2015).

${ }^{59}$ A. Kubica, J. Kowalewski, D. Kruk, and M. Odelius, J. Chem. Phys. 138, 064304 (2013).

${ }^{60}$ S. Khan, A. Kubica-Misztal, D. Kruk, J. Kowalewski, and M. Odelius, J. Chem. Phys. 142, 034304 (2015).

${ }^{61}$ M. R. Pederson and S. N. Khanna, Phys. Rev. B 60, 9566 (1999).

${ }^{62}$ S. Schmitt, P. Jost, and C. van Wüllen, J. Chem. Phys. 134, 194113 (2011).

${ }^{63}$ P. W. Anderson, Phys. Rev. 115, 2 (1959).

${ }^{64}$ W. C. Martin, R. Zalubas, and L. Hagan, "Atomic energy levels - the rareearth elements," National Standard Reference Data Series, NSRDS-NBS 60, National Bureau of Standards, Washington, 1978.

${ }^{65}$ O. Kahn, Molecular Magnetism (VHC, New York, 1993).

${ }^{66}$ J.-J. Girerd, J. Chem. Phys. 79, 1766 (1983).

${ }^{67}$ V. Papaefthymiou, J. J. Girerd, I. Moura, J. J. G. Moura, and E. Münck, J. Am. Chem. Soc. 109, 4703 (1987).

${ }^{68}$ L. Noodleman and E. J. Baerends, J. Am. Chem. Soc. 106, 2316 (1984).

${ }^{69}$ J. D. Rinehart and J. R. Long, Chem. Sci. 2, 2078 (2011). 